Bosch S., Schmidt M. (2019): Auswirkungen neuer Energiesysteme auf die wirtschaftliche Entwicklung - Möglichkeiten eines grünen Kapitalismus. In:

NachhlatigkeitsManagementForum. DOI: https://doi.org/10.1007/s00550-019-00486-w.

\title{
Auswirkungen neuer Energiesysteme auf die wirtschaftliche Entwicklung - Möglichkeiten eines grünen Kapitalismus
}

\author{
Stephan Bosch und Matthias Schmidt
}

\begin{abstract}
Zusammenfassung
Viele Wissenschaftler verknüpfen mit der Energiewende eine wirtschaftliche Neuorientierung der Gesellschaft, in deren Folge es zu einem radikalen Wechsel in den Arbeits- und Lebensverhältnissen sowie den Produktions- und Konsumptionsmustern kommen muss. Sie behaupten, dass die Transformation des Energiesystems hin zu erneuerbaren Energien nicht mehr auf der Logik des Kapitalismus beruhen kann. Ausgehend davon stellt sich die Frage, ob die Abkehr von fossilen Energieträgern tatsächlich und notwendigerweise mit radikalen sozioökonomischen Veränderungen einhergehen muss. Hierzu werden die zentralen Argumente der umwelt- und ressourcenbezogenen Kapitalismuskritik analysiert und im Rahmen einer theoretischen Annäherung dahingehend überprüft, inwieweit sie nachvollziehbar das Ende fossiler Energieträger mit dem Ende des Kapitalismus verknüpfen. Als theoretischer Bezugspunkt dient dabei Joseph Schumpeter's "Theorie der wirtschaftlichen Entwicklung", die mit ihrem Erklärungsansatz der "schöpferischen Zerstörung" auch heute noch bemerkenswerte Einblicke in die Robustheit und Fähigkeiten kapitalistischer Systeme gewährt. Die Studie kommt zu dem Ergebnis, dass die Überwindung der Umwelt- und Ressourcenkrise nicht zwangsläufig durch die Überwindung marktwirtschaftlicher Ansätze erfolgen muss, sondern staatlich begleitete, wettbewerbsorientierte Marktwirtschaften auf Basis regenerativer Energiesysteme eine große ökologische Wirksamkeit entfalten können.
\end{abstract}

\section{Economic development within renewable energy systems - Opportunities for green capitalism}

\begin{abstract}
Many scientists link the conception of an energy transition to an economic reorientation of society, in the wake of which a radical change of the working and living conditions and patterns of production and consumption has to occur. They argue that the transition to a regenerative energy system can no longer be based upon the logic of capitalism. The question arises whether the decline in the use of fossil resources will actually and necessarily induce radical socio-economic changes. For this reason, based on a theoretical approach, the study at hand analyzes the core criticism of capitalism concerning nature and resources with regard to its ability to consistently and logically link the end of fossil energy carriers with the end of capitalism. In this, Joseph Schumpeters' "Theory of Economic Development" serves as the theoretical framework. His theoretical approach entailing the process of "creative destruction" still offers insights into the robustness of capitalist systems and shows that even though fossil fuels and the capitalist system based upon them have given rise to the environmental crisis, surmounting the crisis does not necessarily call for surmounting market-based approaches; rather, market economies based on regenerative energy systems that are competition-oriented and guided by state measures may develop great ecological effectivity.
\end{abstract}

\section{Einleitung}

Die Sorge um die Tragfähigkeit unseres Planeten erfährt seit den Ölkrisen sowie dem Aufkommen der Umweltbewegung eine stetige akademische Zuspitzung in Richtung eines näherkommenden Zusammenbruchs moderner Gesellschaften (Meadows et al. 1972; Daly 1991; Sieferle 1997; Altvater 2005; Schwartzman 2008; Huber 2009; North 2010; Kallis et al. 2009; MartinezAlier 2012). Im Fokus der Debatte steht dabei das Energiesystem, da jede menschliche Tätigkeit Energie benötigt und wirtschaftliches Wachstum immer auch mit einem Anstieg im Energieverbrauch einhergeht (Martinez-Alier 1987; Daly 1995). Die Suche der Menschheit nach neuen Energiequellen ist daher fundamental (Sie- ferle 2001; Solomon und Krishna 2011; 
Rifkin 2013) und äußerst sich in Europa derzeit in der forcierten Substitution fossiler Energieträger durch erneuerbare Energien (Resch et al. 2008; Quaschning 2014). Einen wesentlichen Grund für diese Transformation stellen die negativen Begleiterscheinungen des fossilen Energiesystems dar, die sich im Klimawandel (McCarthy 2015), im Verlust an Biodiversität (Willis und Bhagwat 2009) sowie in den sich intensivierenden Konflikten um die Endlagerung von Atommüll äußern (Rosa et al. 2010).

Mit der Kritik des Club of Rome am menschlichen Umgang mit den Quellen und Senken der Erde sowie der im BrundtlandReport erarbeiteten Politikstrategie einer nachhaltigen Entwicklung, die im Speziellen die Generationengerechtigkeit und die ökologische Vernunft in den Vordergrund der internationalen Entwicklungs- und Umweltpolitik stellte, standen erstmalig nicht mehr nur die quantitativen Merkmale von Energieträgern im Blickpunkt des Interesses, sondern auch ihre qualitativen Eigenschaften (Meadows et al. 1972, S. 72; WCED 1987, S. 20 f.). Jenseits sozio-ökologischer Limits wird die Nutzung von Rohstoffen aber auch durch ihre natürliche Verknappung begrenzt (Meadows et al. 2006, S. $86 \mathrm{ff}$.). Dabei geht es nicht nur um energetische, sondern auch um nicht-energetische Rohstoffe, die in Form von Neodym, Dysprosium und Lithium zu bedeutsamen Ressourcen regenerativer Energiesysteme geworden sind (Viebahn et al. 2015). In der Wissenschaft umstritten ist jedoch, zu welchem Zeitpunkt die Erschöpfung der Vorräte tatsächlich eintreten und eine Umgestaltung des Energiesystems erzwingen wird (Bridge 2010).

García-Olivares und Solé (2015, S. 31) rechnen spätestens 2021 mit Peak Oil und anschließend mit einem raschen Erschöpfen fossiler Energieträger innerhalb weniger Jahrzehnte (vgl. auch Statista 2019). Wie stark derartige Prognosen nicht nur die Kritik an den sozio-materiellen Verhältnissen marktwirtschaftlicher Ordnungen befeuern, sondern zugleich apokalyptische Ängste angesichts des Versiegens fossiler Energiequellen stimulieren, beschreibt Swyngedouw (2010, S. 219) eindrücklich. Bemerkenswert in diesem Zusammenhang ist, dass die Europäische Kommission in ihrer Strategie zur Reduktion der Treibhausgasemissionen die bestehenden sozio-materiellen Verhältnisse keineswegs in Frage stellt, sondern von einer kosteneffizienten Transformation des Energiesektors ausgeht (European Commission 2018). Damit rückt die europäische Politik die fossilen Energieträger ins Zentrum der Kritik. Im Gegensatz dazu bezieht sich die Kritik politisch linksorientierter Akteure nicht nur auf die fossilen Energieträger, sondern stellt das gesamte ökonomische System infrage, das auf dem bislang stabilen Fundament fossiler Energien funktionieren konnte - den Kapitalismus. Nach Huber (2009, S. 105) wird die Beziehung des Kapitalismus zur Natur entscheidend durch seine starke Abhängigkeit von fossilen Energieträgern bestimmt. Die Logik von stetigem Wachstum basiere allein auf den einzigartigen Eigenschaften von Kohle, Erdgas sowie Öl und könne nicht auf ein regeneratives Energiesystem übertragen werden. Dieser Argumentation folgend würde mit dem Ende fossiler Energieträger das Ende des kapitalistischen Systems einhergehen (Altvater 2007; Huber 2013; Garcia-Olivares und Sole 2015; Kinder 2016; Buch-Hansen 2018).

Wenngleich Kenis und Lievens (2016) es als wünschenswert erachten, die Neugestaltung des Energiesystems mit einer Neuordnung der sozio-materiellen Beziehungen zu verknüpfen, so sehen sie in einer Transformation des Energiesystems in Richtung erneuerbarer Energien nicht nur eine ausreichende Strategie zur Bewältigung der Umweltkrise, sondern auch die Gelegenheit für den Kapitalismus fortzubestehen. Auch Harriss-White und Harriss (2007, S. 76) betonen, dass erneuerbare Energien problemlos in die kapitalistische Logik von Markt und Massenproduktion eingebettet werden können. Selbst Klimawandel und Klimaschutz werden den Mechanismen des Marktes sowie den Kompetenzen von GeoEngineering überlassen, ohne dass dabei die sozio-materiellen Verhältnisse grundlegend infrage gestellt werden (McCarthy 2015, S. 2490 f.). Dies erlaube ein Umschiffen der ökologischen Krise und eine Fortführung der Akkumulation im Rahmen eines ökologisch stärker angepassten kapitalistischen Systems. Mathews (2011) und Böhm et al. (2012) halten eine Transformation des Kapitalismus in Richtung eines „Green New Deal“ ebenfalls für möglich, wobei sie wie Brand (2016) darin keinen Ansatz zur Lösung sozialer Probleme und globaler Ungleichheiten erkennen können. Gerade marxistisch orientierte Umweltforscher lehnen einen grün-gemäßigten Kapitalismus daher strikt ab (Harris 2010, S. 76), sehen sie in der kapitalistischen Ordnung doch den Ursprung der Zerstörung aller sozialen und umweltbezogenen Verhältnisse (Polanyi 1957; Harris 2013).

Wir haben es folglich mit zwei wesentlichen Dimensionen der Kritik an kapitalistisch ausgerichteten regenerativen Energiesystemen zu tun. Die erste Dimension verneint

die Möglichkeit eines grünen Kapitalismus und beinhaltet die Annahme, dass die kapitalistische Ordnung, die sich maßgeblich aus den sozio-materiellen Eigenschaften fossiler Energieträger heraus entwickelt hat, nicht auf die soziomaterielle Logik regenerativer Energiesysteme übertragen werden kann und eine Abkehr von fossilen Energieträgern so zwangsläufig mit einer wirtschaftlichen Ordnung jenseits des Kapitalismus einhergehen muss. Die zweite Dimension bejaht die Möglichkeit eines grünen Kapitalismus, lehnt ihn aber aus Gründen der sozialen Gerechtigkeit sowie aufgrund der 
ökologisch-materiellen Limits von Massenmärkten ab und favorisiert daher einen Aufbau regenerativer Energiesysteme innerhalb eines sozio-materiell vollkommen transformierten gesellschaftlichen Rahmens.

Unser zentrales Anliegen ist es, die folgenden Annahmen, die diesen beiden Dimensionen der Kritik zu Grunde liegen, zu überprüfen:

- die Abkehr von fossilen Energieträgern geht zwangsläufig mit einem Rückgang in der Produktivität einher (vgl. Altvater 2007; Huber 2009; North 2010)

- die Umweltkrise ist ein Beleg für das Versagen und die fehlende Zukunftsfähigkeit des Kapitalismus (vgl. Harvey 2011; Kallis 2011; Huber 2013; Buch-Hansen 2018) Innovation ist ein Phänomen, dass sich rein auf das Technologische und Vorstellbare bezieht und eine Gesellschaft nicht in Richtung Nachhaltigkeit bringen kann (vgl. Harriss-White und Harriss

- 2007; Kallis 2011; McCarthy 2015)

- die Möglichkeiten eines grünen Kapitalismus sind stark begrenzt (vgl. Castree 2010; Böhm et al. 2012; Harris 2013; McCarthy 2015; Brand 2016)

- eine Differenzierung zwischen den verschiedenen Formen kapitalistischer Systeme ist unnötig, denn jegliche Form des Kapitalismus gefährdet den Aufbau eines nachhaltigen Gesellschaftssystems (vgl. Kallis 2011; Mathews 2011; Huber 2013; Kinder 2016)

- der Aufbau regenerativer Energiesysteme im Rahmen einer kapitalistischen Ordnung führt zu einer weiteren Vertiefung sozialer Ungleichheiten (vgl. Aitken 2010; Cowell 2010; Huber 2013)

Der Aufsatz ist folgendermaßen strukturiert: Nachdem wir in Kap. 2 auf die in die Krise geratene enge Beziehung zwischen fossiler Energie und Kapitalismus eingegangen sind, wollen wir in Kap. 3 auf die erste Dimension der Kritik eingehen. Dabei gehen wir von der These aus, dass der Kapitalismus ausreichend robust sowie dazu fähig ist, die gegenwärtige Umweltkrise, die zu erheblichen Veränderungen in der Rohstoff und Energiebasis führen wird, zu überwinden und seine Produktivität aufrechtzuerhalten. Mit Blick auf die zweite Dimension der Kritik gehen wir in Kap. 4 von der These aus, dass die prinzipielle Ablehnung eines grünen Kapitalismus innovative Ansätze zur Bewältigung der Umweltkrise übersieht und damit kontraproduktiv ist. Es besteht jedoch die Vermutung, dass Innovationen, um zielgerichtet sein zu können, einer politischen Lenkung bedürfen. Daher überprüfen wir in Kap. 5 die These, dass der Einsatz marktorientierter Strategien zur Bewältigung der Umweltkrise nur dann gelingen kann, wenn er seitens des Staates gestützt und kontrolliert wird. In diesem Zusammenhang werden auch die sozialen Auswirkungen marktbasierter regenerativer Energiesysteme näher untersucht, wobei wir von der These ausgehen, dass die sozialen Ungleichheiten im Rahmen der Energieversorgung mit der Energiewende reduziert werden können.

Den theoretischen Hintergrund der Thesen bildet die Erkenntnis, dass Innovationen einerseits die Fähigkeit besitzen, (energie-)wirtschaftliche Prozesse radikal zu transformieren, Innovationen andererseits mit einer größeren Häufigkeit und Wahrscheinlichkeit in einer kapitalistischen, als in alternativen gesellschaftlichen Ordnungen auftreten. Die wissenschaftlichen Grundlagen hierzu hat Joseph Schumpeter geliefert, der im Rahmen seiner „Theorie der wirtschaftlichen Entwicklung" (Schumpeter 1912, 1994) großen Wert auf die Unterscheidung zwischen einem wettbewerbsorientierten Konkurrenzkapitalismus sowie einem vertrusteten Kapitalismus gelegt hat. Bei Ersterem gilt den fruchtbaren zerstörerischen Impulsen, die von enthusiastisch voranpreschenden, risikobereiten sowie problemlösungsorientierten Unternehmern ausgehen, ein besonderes Augenmerk: "This process of Creative Destruction is the essential fact about capitalism" (Schumpeter 2009, S. 42 f.). Das bereits von Marx und Engels erarbeitete sowie kritisch betrachtete Konzept des Konkurrenzkapitalismus (Angebots-Polypol: Bezeichnung in den nicht-marxistischen Wirtschaftswissenschaften), das von einem gesellschaftlich unkoordinierten Kampf zwischen einer Vielzahl an profitorientierten Unternehmern ausgeht (Duthel 2013, S. 237), wird im Folgenden zum Leitkonzept erhoben. Hierdurch kann die ursprüngliche, in den Diskursen zur Nachhaltigkeit jedoch kaum mehr transportierte Idee des innovationsgetriebenen Kapitalismus wieder freigelegt (Marques 2008, S. 288) und hinsichtlich ihres potenziellen Beitrags zur Bewältigung der Umweltkrise untersucht werden. Nach Überprüfung der zentralen Thesen werden in Kap. 6 die Ergebnisse der Studie zusammengefasst.

\section{FossileEnergienundKapitalismusinder Krise}

Veränderungen in der Bedeutung von Energieträgern haben immer auch zu umfassenden ökonomischen und sozialen Transformationen geführt (Bridge et al. 2013, S. 331). Energie ist damit ein zentrales Leitmotiv gesellschaftlicher Entwicklung. Beispielhaft hierfür steht die erhebliche Stei-gerung der Produktivität während der Industrialisierung, die nicht ohne die revolutionäre Veränderung des Energieregimes in Richtung fossiler Energieträger zu erklären ist (Osterhammel 
2011, S. 916). Wirtschaftliches Wachstum geht seitdem mit einem steigenden Verbrauch an endlichen energetischen sowie nicht-energetischen Rohstoffen einher (Altvater 2005, S. 6 ff.). Folglich ist die wirtschaftliche Entwicklung immer im Zusammenhang mit dem Energiesystem sowie die in ihm zirkulierenden Stoffströme zu sehen (Meadows et al. 2006). Die Ausrichtung des Kapitalismus nach industrieller Skalierung und quantitativem Wachstum ist jedoch kritisch zu betrachten (Mathews 2011, S. 872), da sie ein Ende finden wird, sobald die begrenzten Kapazitäten der Erde durch exponentielles Wachstum von Bevölkerung und Wirtschaft erschöpft sein werden (Daly 1995). Doch nicht nur die Endlichkeit von Energieträgern, auch die Häufung meteorologischer Extremereignisse, massenhaftes Artensterben und der Anstieg des Meeresspiegels stellen ein Hindernis für stabiles wirtschaftliches Wachstum dar (McCarthy 2015, S. 2490). Der Club of Rome verdeutlicht in seinen Szenarien zur Entwicklung des Zustandes der Welt, dass der Wohlstand nur dann aufrechtzuerhalten ist, wenn ein radikales Umdenken im Hinblick auf die Bewertung von Wachstum greift (Meadows et al. 2006, S. 248 ff.) und die ökologischen Kosten seitens der global agierenden Unternehmen nicht mehr externalisiert werden (Chisholm 1990; UBA 2016).

Georgescu-Roegen $(1986$, S. 16) betont, dass die heraufziehende Energiekrise kaum verstanden werden kann, wenn nicht die Thermodynamik ins Zentrum der Betrachtung gerückt wird. Im Wesentlichen geht es dabei um die Frage des „Energy Return On Investment" (EROI) bzw. um das Verhältnis zwischen Energieaufwand und -ertrag (Bardi 2009, S. 323). Entsprechend den grundlegenden Ausführungen zur Gewinnung von Arbeit aus Wärme seitens Clausius (1850, S. 368 ff.) wird der EROI im historischen Verlauf schlechter. Hieraus lässt sich ableiten, dass die Erschließung und Nutzung begrenzter Vorräte energetisch immer ineffizienter wird und der kapitalistischen Produktion so unweigerlich eine Grenze setzt (Murphy und Hall2011). Dies lässt sich dadurch erklären, dass dem ökonomischen System auf der Inputseite zwar wertvolle Energie, mit einer sehr geringen Entropie, zuströmt (z. B. Rohöl aus einer Ölquelle). Auf der Outputseite gibt es jedoch wertlose Energie, mit einer hohen Entropie, wieder an das Ökosystem ab (z. B. Abwärme von Kraftwerken). Entsprechend des 1. Hauptsatzes der Thermodynamik, der besagt, dass die Energie im Universum konstant bleibt, geht beim Durchgang der Energie durch den ökonomischen Prozess zwar keine Energie verloren. Doch da gleichzeitig die Entropie gegen ein Maximum strebt und die transformierte Energie, bspw. in Form von hochentropischen Verbrennungsrückständen, nicht mehr verwertbar ist, erwächst dennoch ein rohstoffliches Limit (Georgescu-Roegen 1971, S. 3 ff.).

Dieses von den Ökologischen Ökonomen (Martinez-Alier 2012) hervorgehobene Limit von Stoffströmen innerhalb einer "vollen Welt“ (Daly 2005) und die darauf basierende Forderung nach einem „Steady State“ (Daly 1991) sind jedoch selbst nach marxistischer Auffassung überbewertet. So sollte Energie nicht rein als materielle Ressource betrachtet werden, sondern als ein Konzept sozialer Beziehungen, geprägt von Macht und sozialökologischem Wandel (Huber 2009, S. 106 ff.). Bridge (2010, S. 524 f.) sieht speziell in den scharfen Debatten um „Peak Oil“ die ökologisch motivierte Ankündigung einer neuen Energieordnung, in der sich die modernen Industriestaaten von ihrer Ölabhängigkeit befreien würden. Neomarxistische Gruppierungen wollen im Ende des Ölzeitalters gar eine apokalyptische Zeitenwende erkennen, bei der sich die Natur an der ökologischen Überheblichkeit des Kapitalismus rächen wird. Nach Bettini und Karaliotas (2013, S. 331 f.) erlangt die Peak Oil-Narration dadurch eine Symbolik, die weit über die mathematischen Berechnungen zur Knappheit fossiler Energieträger hinausreicht und zur generellen Kritik an einem System erweitert wird, das ausschließlich an Wachstum orientiert ist. Die Chance eines post-fossilen Kapitalismus sieht McCarthy (2015, S. 2496), der dies nicht willkommenheißt, in der Kommodifizierung von Wind, Sonnenlicht, Erdwärme und Wellen. Damit würde die Natur erneut in den Kreislauf des Kapitals hineingezogen werden. Van den Bergh (2011) erkennt hierin einen gangbaren Weg und empfindet die Kritik an Marktwirtschaft und Kapitalismus, die sich einseitig an der Problematisierung von Wachstum abarbeite und dabei keinerlei realisierbare Alternativen aufzeige, als zu radikal. Van den Bergh $(2011$, S. 881) präferiert das sog. "a-growth-Konzept", dass von einer neutralen Position ge- genüber Wachstum ausgeht und soziale sowie ökologische Nachhaltigkeit über Preispolitik, Umweltabkommen und Bildungsinitiativen herzustellen versucht.

Die Kommodifizierung von Natur wird seitens der wachstumskritischen Degrowth-Bewegung (Degrowth= Postwachstum) abgelehnt, da sich beim Vergleich des auf Vorschriften basierenden Montreal-Protokolls (Ozon) mit dem handelsbasierten Kyoto-Protokoll (Klimawandel) die größere Wirksamkeit regulativer Maßnahmen gezeigt habe (Kallis 2011, S. 877). Zudem spricht North (2010, S. 586) in Bezug auf die Möglichkeiten des Marktes von stupider Technikgläubigkeit neoliberaler Enthusiasten, die irrtümlicherweise davon überzeugt sind, dass die schöpferische Zerstörung dazu ausreiche, den gesellschaftlichen Herausforderungen Peak Oil und Klimakrise zu begegnen. Sarkar und Kern (2008) spitzen die Möglichkeiten der Weltgemeinschaft im Rahmen ihrer Kapitalismuskritik auf die Optionen „Ökosozialismus oder Barbarei" zu. DerKapitalismus wird durch diese Rhetorik zum Feindbild stilisiert, da er aufgrund der Ausbeutung natürlicher Ressourcen einerseits die Ursache der Umweltkrise darstellt (Daly 2005, S. 100 ff.), andererseits zur Krisenbewältigung keinen geeigneten gesellschaftlichen Rahmen bietet (Kallis et al. 2009, S. 22). Deshalb wird für den 
Aufbau einer Ökonomie geworben, die jenseits von obsessivem wirtschaftlichem Wachstum liegt (Buch-Hansen 2018, S. 157). Inwieweit dies notwendig erscheint, wird im Folgenden untersucht.

\section{Robustheit desKapitalismus}

\subsection{Räumlich-zeitlicheKompression}

Altvater (2007), Huber (2009) und North (2010) behaupten, dass sich der Kapitalismus allein auf Basis fossiler Energieträger ausbreiten kann, da durch die globale Transportierbarkeit von Öl, Gas und Kohle unternehmerische Tätigkeiten nicht an lokal verfügbare Energieressourcen gebunden sind, sondern eine globale Reichweite besitzen. Zudem unterliegen fossile Energieträger keinen tageszeitlichen und saisonalen Schwankungen. Transportierbarkeit und Grundlastfähigkeit führen so zur räumlich-zeitlichen Kompression (Harvey 1996, S. 242 ff.), da Produkte in immer kürzeren Zeitintervallen hervorgebracht werden. In dieser Logik würde mit der Limitierung der fossilen Rohstoffbasis das Ende des kapitalistischen Systems einhergehen.

Dass der Energiefluss in einem solarbasierten Energiesystem schwer kontrollierbar ist (Georgescu-Roegen 1971, S. 303 f.), bleibt unbestritten. Es handelt sich bei den meisten Formen erneuerbarer Energien um intermittierende Quellen, deren Beiträge zum Energiemix den Rhythmen von Sonne, Wind, Niederschlag und Gezeiten unterliegen (Fares 2015). Die Anpassung der Energieproduktion an den Bedarf, eine Grundvoraussetzung für kontinuierliches Wirtschaftswachstum, wird damit zu einer großen Herausforderung. Was Altvater (2007), Huber (2009) und North (2010) nicht in ihre Überlegungen miteinbeziehen, sind die zahlreichen technologischen Innovationen zur Stabilisierung regenerativer Energiesysteme. So stehen mit Biomasse und Erdwärme zwei grundlastfähige Energieträger zur Verfügung (Matek und Gawell 2015, S. 101), die im Rahmen von regenerativen Kombikraftwerken die witterungsabhängigen Energiequellen Sonne und Wind stützen (Palensky und Dietrich 2011, S. 381; Ramchurn et al. 2011, S. 5). Bedeutend sind zudem die innovativen Technologien zur Energiespeicherung (McCarthy 2015, S. 2491), von denen bislang jedoch nur wenige die industrielle Reife erlangt haben. Dabei werden mechanische, chemische, elektrische und thermische Speicherarten unterschieden (Hadjipaschalis et al. 2009, S. $1514 \mathrm{ff}$.). Vielversprechend sind Druckluft-und Pumpspeicherkraftwerke, mit Wirkungsgraden von bis zu $80 \%$ (Anagnostopoulos und Papantonis 2008, S. 1685). Geforscht wird aber auch an der Umwandlung von überschüssigem Regenerativstrom in Methan oder Wasserstoff (Jensen et al. 2007, S. 3253), wodurch eine bidirektionale Verknüpfung von Stromsowie Gasnetz und die Transportierbarkeit sowie Grundlastfähigkeit innerhalb großer Raumeinheiten möglich wird. Die räumlich-zeitliche Verfügbarkeit kann zudem durch den Ausbau und die Kapazitätserweiterung von Stromtrassen erhöht werden (Walter und Bosch 2013). Je größer der Raum ist, innerhalb dessen Regenerativstrom intelligent organisiert und über verlustarme Stromleitungen zugeteilt wird, desto höher ist die Wahrscheinlichkeit, dass zu einem bestimmten Zeitpunkt tatsächlich Energie zur Verfügung steht (McCarthy 2015, S. 2492). Dabei spielt der Ausbau von HochspannungsGleichstrom-Übertragungsleitungen eine entscheidende Rolle, da hierdurch die Transportierbarkeit von Regenerativstrom über weite Strecken hinweg wirtschaftlich wird. Harriss-White und Harriss (2007, S. 75) haben darauf hingewiesen, dass die bestehenden Stromnetze, die aus einer monopolitischen Logik heraus entwickelt wurden, veraltet und nicht dazu geeignet sind, dezentral erzeugten Strom mit starken Fluktuationen zu integrieren. Diese Defizite werden aber sukzessive aufgearbeitet. So erhält der windschwache, industriestarke Süden Deutschlands einen direkten Zugang zu den großen Windenergie-Offshore- Potenzialen im Norden sowie zu den Speicherkraftwerken in Skandinavien (vgl. Abb. 1). Die Möglichkeiten des interkontinentalen Stromtransports aus erneuerbaren Quellen wurden im Rahmen der Studien zum Desertec-Projekt grundlegend erörtert (German Aerospace Center (DLR) 2006). Grossmann et al. (2014) diskutieren sogar die methodischen Grundlagen zu einem globalen Stromverbund allein auf Basis von Solarenergie.

Energiespeicherung und Stromnetzausbau werden folglich die derzeit noch bestehenden räumlich-zeitlichen Einschränkungen regenerativer Energiesysteme aufheben und dem postfossilen Kapitalismus dadurch eine große Robustheit verleihen. Beide Bereiche sind jedoch nicht isoliert voneinander, sondern werden über Smart Grids koordiniert. Solomon und Krishna (2011, S. 7429) betonen, dass sich Smart Grids hervorragend zur Implementierung marktbasierter Ansätze eignen und so künftig von einem innovationsgetriebenen Massenmarkt für Energieeffizienz-Technologien auszugehen ist. Smart Grids eröffnen auch die Möglichkeit, die massenhafte Produktion von neuen Technologien, die die regenerativen Quellen in Energie wandeln, nicht mehr auf Basis fossiler Energie, sondern mittels erneuerbarer Energie zu gestalten. Zwar basierte die Herstellung der ersten Generationen regenerativer Technologien auf fossiler Energie, die Möglichkeiten zur Energiespeicherung, das schier unbegrenzte Energiepotenzial einer solar- 
Abb. 1 Ausbau der Hochspannungs-Gleichstrom-Übertragung in Deutschland - räumlichzeitliche Kompression in regenerativen Energiesystemen

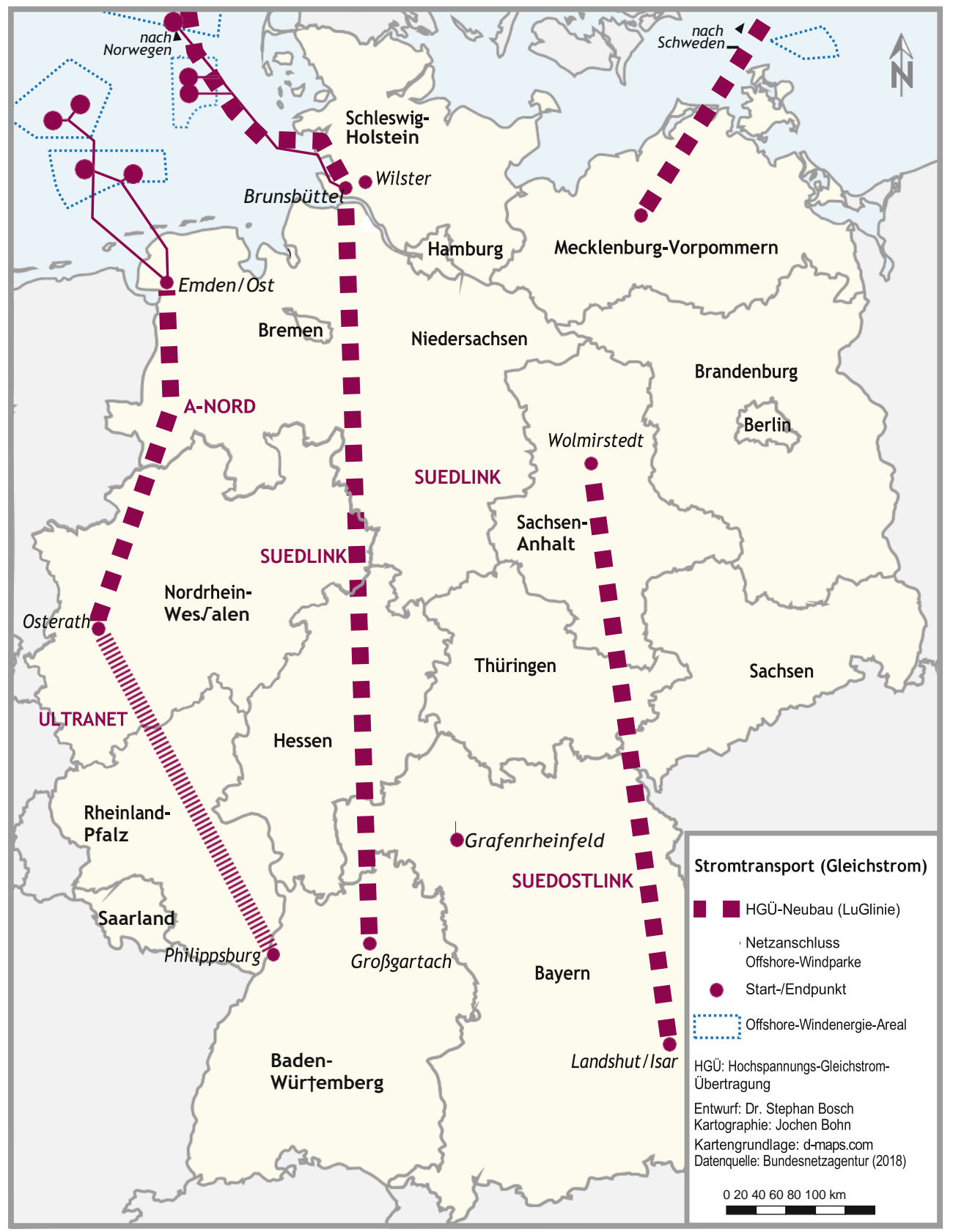

basierten Wirtschaft und die Koordination beider Aspek te über Smart Grids werden künftig jedoch eine zeitlich flexible Bereitstellung von regenerativer Energie an allen Produktionsstandorten gewährleisten. Eine Steigerung der Produktivität sowie stabiles wirtschaftliches Wachstum sind damit auch im Rahmen eines regenerativen Energiesystems möglich.

\subsection{Krise als Element kapitalistischer Ordnung}

Harvey (2011, S. 6) erläutert, dass alles, was die Zirkulation und Akkumulation von Kapital blockiert, zu einer Bedrohung für das kapitalistische System werden und eine fundamentale Krise herbeiführen kann. Die Endlichkeit fossiler Energieträger stellt solch eine Krise dar (McCarthy 2015, S. 2490). Altvater (2007, S. 54) ist der Überzeugung, dass der Kapitalismus diese Krise nicht bewältigen kann; der technologische Fortschritt müsse folglich in einen nicht- fossilen, nicht-kapitalistischen Rahmen eingebettet werden. Auch Kallis (2011, S. 875) betont, dass die Annäherung an einen Steady State - vgl. Daly (1991, 
2005) - die institutionellen Rahmenbedingungen von Eigentum, Arbeit, Kreditwesen und Verteilung derart transformieren wird, dass am Ende kein Kapitalismus mehr identifizierbar sein wird. Schumpeter weist im Hinblick auf die von Kallis bezweifelte institutionelle Robustheit des Kapitalismus darauf hin, dass sich Fortschritt gerade im Auf und Ab industrieller Entwicklungen, die das Ergebnis erfolgreicher und den Wettbewerb verstärkender Innovationen sind, entfaltet (Herzog und Honneth 2016, S. 44, 55). Da Krisen also einen immanenten Bestandteil des kapitalistischen Systems bilden, ist eine durch den kapitalistischen Prozess mit verursachte Umwelt- und Ressourcenkrise kein hinreichender Beleg für einen möglichen Niedergang kapitalistischer Ordnung. Sie könnte sogar als Beleg für einen Konjunkturzyklus angesehen werden, wenn sie als eine Phase der Depression zwischen dem vergehenden fossilen Zeitalter und dem aufkommenden Zeitalter regenerativer Systeme verstanden wird. Böhm et al. (2012, S. 1619) und McCarthy (2015, S. 2495) bestätigen, dass der Kapitalismus selbst fundamentale Krisen überstehen und ausgehend davon immer weiter expandieren konnte. In Bezug auf die Umweltkrise erkennen auch Harriss-White und Harriss $(2007$, S. 73) an, dass der Ausbau erneuerbarer Energien das Potenzial besitzt, eine neue Form des Kapitalismus hervorzubringen, die sich durch eine wesentlich geringere materielle Verschwendung auszeichnet. Sogar Altvater (2007, S. 48) weist darauf hin, dass die externalisierten Auswirkungen von Produktion und Konsumption auf die Natur für Unternehmen dann an Relevanz gewinnen, wenn sie die Profitabilität und Akkumulation beeinträchtigen. Ist dies der Fall, können Umweltprobleme sowie entsprechende Lösungen sogar zu einem Teil der kapitalistischen Logik werden. Bettini und Karaliotas (2013, S. 337) betonen diesbezüglich, dass innerhalb neoliberaler Weltanschauung der Vorwurf einer kapitalistisch bedingten Umweltkrise keineswegs zu Selbstzweifel führt. Vielmehr rücke hierdurch die profitable Vermarktung adäquater Lösungsansätze im Bereich Ressourceneffizienz und Umweltschutz in den Blickpunkt des ökonomischen Interesses. Politisches Handeln werde dabei durch marktund technologieorientiertes Management substituiert. Nicht zuletzt sieht McCarthy (2015, S. 2495) in einer groß angelegten Transformation des Energiesystems, mit Ausgaben von mehreren Billionen Dollar, dem Aufbau neuer Produktionsstätten und Infrastrukturen sowie der Schaffung neuer Absatzmärkte, die Gelegenheit für die kapitalistische Akkumulation, sich auch im Rahmen einer post-fossilen Gesellschaft zu entfalten. Solomon und Krishna (2011, S. 7430) sind der Überzeugung, dass hierzu noch nicht einmal technologische Durchbrüche notwendig sind, da die benötigten Technologien zur Umstellung der Gesellschaft auf Energieeffizienz bereits ausgereift und kosteneffizient sind.

Kallis (2011, S. 878) räumt ein, dass der Kapitalismus eine große Robustheit besitzt. Dennoch sieht er in der Krise die Gelegenheit, bislang unverrückbar erscheinende, blockierende soziale und politische Lock-ins, die in die Krise geführt haben, aufzubrechen. Er sieht die Fähigkeit zur sozialen und politischen Transformation dabei nicht in den Eigenschaften des Marktes, sondern in einer an Degrowth bzw. Postwachstum orientierten Ordnung. Kallis hat sicherlich Recht damit, dass der Markt schwer zu kontrollieren ist und eine gezielte Transformation in Richtung Nachhaltigkeit dadurch erschwert wird. Seine Kritik bezieht sich jedoch allein auf jene Form des Kapitalismus, die Schumpeter als vertrusteten Kapitalismus bezeichnet und in der Tat zu Lock-in-Effekten führt (Schumpeter 1912, 1994). Auf den Konkurrenzkapitalismus kann diese Kritik aber nicht bezogen werden, denn gerade aus ihm gehen die Basisinnovationen hervor, die zu den von Kallis als so fruchtbar beschrieben revolutionären Krisen führen und überhaupt erst die Chance für alternative gesellschaftliche Verhältnisse - jedoch innerhalb der kapitalistischen Ordnung - eröffnen. Zwar können Innovationen auch außerhalb von Wettbewerb und Markt entstehen, jedoch nicht in der erforderliche Dichte und Stärke, da Wachstum der wichtigste Treiber für Innovationen ist, wie Wangler (2013, S. 213) anmerkt. Umgekehrt führt ein kontinuierlicher Innovationsprozess wiederum zu Wachstum, das die bestehenden gesellschaftlichen Verhältnisse revolutionieren kann, wie Schumpeter (1912) feststellt. Dabei kommt es zu einer neuen Kombination vorhandener Produktionsmittel in neuen Produktionsstätten, wobei neue Güter, neue Methoden und neue Märkte hervorgebracht werden. Produktive Ressourcen werden dabei den bisher unerprobten Verwendungen zugeführt und aus Verwendungen, denen sie bisher gedient haben, abgezogen (Herzog und Honneth 2016, S. 127). Was Kallis (2011, S. 877) in Bezug auf die ökologische Innovationskraft des Kapitalismus als technologischen Optimismus bezeichnet, ist im Kontext von Schumpeters Konkurrenzkapitalismus daher technologischer Realismus.

Zweifelsohne sind Innovationsschübe auch in bereits etablierten Unternehmen denkbar (Herzog und Honneth 2016, S. 136), wodurch der vertrustete Kapitalismus, mit seinen ökologisch bedenklichen Strukturen, weiter aufrechterhalten werden kann. Beispielhaft steht hierfür die Innovation "Carbon Dioxide Capture and Storage“, mittels derer der ökologische Impact des emissionsstarken Kohlestroms verringert wird (Benson und Orr 2008, S. 303). Technologischer Fortschritt kann folglich das bestehende, für die Umweltkrise verantwortliche Politik- und Wirtschaftssystem verstetigen (Bettini und Karaliotas 2013, S. 337). Entscheidend ist für Schumpeter jedoch der Konkurrenzkapitalismus, der sich durch das aggressive wirtschaftliche Auftreten neuer, innovativer Unternehmen auszeichnet und das Establishment ökonomisch herausfordert (Herzog und Honneth 2016, S. 136). Wettbewerb ist bei der Bewältigung der Umweltkrise daher zentral. Insofern ist die Maxime des Konzepts "Solidarische Ökonomie“, das Prinzip der Konkurrenz zu überwinden und sich von der Logik des 
Marktes zu emanzipieren (Embshoff und Giegold 2008, S. 12), kontraproduktiv. Durch den Verzicht auf Wettbewerb können verkrustete Wirtschaftsstrukturen nicht aufgebrochen werden und bleiben so zum Schaden der Umwelt bestehen. Nicht zuletzt war und ist es die Strategie der großen Energieversorger, möglichst lange an dem fossil-nuklearen Kraftwerkspark festzuhalten und alternative Versorgungskonzepte zu unterdrücken (Gawel et al. 2012). Eine radikale Transformation kann daher nicht aus den bestehenden Versorgungsstrukturen und den alten Unternehmen emporsteigen, wie Schumpeter feststellt (Herzog und Honneth 2016, S. 134 ff.). Innovative Marketingstrategien kleinerer und mittlerer Unternehmen, die zur Verdrängung schwerfälliger großer Konzerne führen, spielen gerade im Bereich erneuerbarer Energien eine wesentliche Rolle (Walsh 2012, S. 32). Wettbewerb und krisenhafte Situationen sind dabei zentral und lassen sich nicht ohne Weiteres ersetzen, denn sie verhelfen Nischeninnovationen zum Durchbruch und zur Transformation sozio-technologischer Regime (Geels 2011, S. 28).

\section{MöglichkeitendesgrünenKapitalismus}

Schumpeter vertritt die Auffassung, dass Innovationen die Folge des kapitalistischen Unternehmergeistes sind, nicht umgekehrt (Herzog und Honneth 2016, S. 151). Technologischer und gesellschaftlicher Fortschritt sind demnach keine unabhängigen Größen, die aus dem Nichts emporsteigen, sondern sie entstehen aus der Logik des kapitalistischen Prozesses. Meadows et al. (2006) anerkennen, dass Innovationen die Grenzen des Wachstums verschieben und den Lebensstandard mit einem immer geringer werdenden Rohstoff- und Energieverbrauch aufrechterhalten können. Das Energiesystem krankt aber immer noch daran, dass verbrauchte sowie noch nicht erschlossene Ressourcen auf Basis nichtregenerativer Energie (wieder-)gewonnen werden (Schwartzman 2008, S. 43 ff.) und die kapitalistische Produktion dadurch energetisch immer ineffizienter wird (Murphy und Hall 2011). Die Meisterung der Energiekrise verlangt daher nach der Berücksichtigung thermodynamischer Grundlagen (Georgescu-Roegen 1986; Martinez-Alier 1987). Im Ausbau erneuerbarer Energien sehen Harriss-White und Harriss (2007, S. 76) die Möglichkeit, die Entropieerzeugung einzudämmen. Kaberger und Mansson (2001, S. 176 f.) haben hierzu aufgezeigt, dass innovative ressourcenschonende Stoffkreisläufe möglich und wirtschaftlich sind, wenn sie auf der Nutzung der nicht erschöpfbaren solaren Strahlungsenergie basieren. Vielversprechend dabei ist, dass die Nutzung der Solarenergie im Zuge von Forschung und Entwicklung immer effizienter und lukrativer wird (Schmid 2016). Zudem erlaubt ihr unerschöpfliches Potenzial die Erschließung materieller Ressourcen selbst aus Lagerstätten mit sehr geringen Rohstoffdichten. Durch den Einsatz von Solarenergie kann Entropie lokal sogar verringert werden (Ebeling et al. 1998, S. 42 f.; Kranert und Cord-Landwehr 2010, S. 77), so wie das bei der Nutzung der Abwärme solarthermischer Kraftwerke zur Meerwasserentsalzung der Fall ist (German Aerospace Center (DLR) 2007).

Innovation geht aber über das rein Technologische - vgl. Roddis (2018) - hinaus und kann auch soziale sowie institutionelle Aspekte umfassen (Arentsen und Bellekom 2014, S. 3 f.). Beispielhaft steht hierfür der Mobilitätssektor, der mit seinen Schadstoffemissionen erheblich zur Umweltkrise beigetragen hat. Innovationen haben im Bereich Mobilität zu neuen Formen des Güterund Personentransports geführt. Am Beispiel von "Car Sharing" als innovativem Lebensstil (Prettenthaler und Steininger 1999, S. 443) oder im Kontext von „bicycle-sharing schemes“ in urbanen Räumen (Midgley 2011, S. 2) wird dies deutlich. Auch die Geschichte des Ozonlochs kann als ein Beleg für die Fähigkeiten des bestehenden Wirtschaftssystems zur Korrektur massiver Grenzüberschreitungen verstanden werden, wobei es nicht nur um technologische, sondern auch um organisatorische sowie institutionelle Verbesserungen ging. Ganz im Sinne Schumpeters führen Meadows et al. (2006, S. 204 f.)

„kreative Köpfe der Industrie“, die ihre Innovationen aufeinander abgestimmt haben, als entscheidende Determinante der Problemlösung an. Mittels der drei Innovationsschübe "bessere Isolierung", "weniger giftige Ersatzstoffe" und „emissionsfreie alternative Stoffe“ wird es gelingen, bis zur Mitte des 21. Jahrhunderts die ursprüngliche Dichte der Ozonschicht wiederherzustellen, und dies, ohne die bestehende wirtschaftliche Ordnung aushebeln zu müssen.

Van den Bergh (2011) kritisiert, dass die Anhänger der Degrowth-Bewegung, die die bestehende wirtschaftliche Ordnung überwinden wollen, nicht dazu in der Lage sind, wirtschaftliche Alternativen zu konkretisieren und stets in der Kritik steckenbleiben. Umgekehrt bestreitet Kallis (2011, S. 874) die Möglichkeit einer entmaterialisierten Wirtschaft, da die Effizienzgewinne durch den steigenden Konsum überkompensiert werden. Wenngleich die Entmaterialisierung seiner Aussage nach einem Wunder gleichkommen würde, so arbeiten Forscher dennoch daran, die materialisierte Wirtschaft ökologisch auszurichten (Baccini und Brunner 2012). Dabei geht es darum, Umweltschutz völlig neu zu definieren, denn Umweltschutz wird heutzutage auf den Versuch verengt, Prozesse und Produkte weniger schädlich zu gestalten (Mulhall und Braungart 2010). Dies birgt aufgrund der potenziellen Entstehung eines neuen Massenmarktes für 
umweltfreundlichere und effizientere Produkte jedoch die Gefahr, dass sich die ungewollten Wirkungen sogar noch verstärken. Alcott (2005) weist diesbezüglich auf das Jevons paradox hin, das besagt, dass es ein großer Irrtum ist zu glauben, technologische Innovationen würden den Konsum von Ressourcen reduzieren. Polimeni et al. (2015) bringen als Beispiel die "Green Revolution", denn die beachtliche Steigerung in der Flächeneffizienz der Nahrungsmittelproduktion konnte keineswegs das Problem des Hungers und Flächenverbrauchs mindern, da die Bevölkerungszahl in der Folge stark angewachsen ist (Rebound-Effekt). Ein Massenmarkt an umweltverträglicheren Produkten würde ebenso wieder zu einem massenhaften Giftund Abfallaufkommen führen, wobei die entsorgten Rohstoffe zudem kaum wiederverwertet würden. Die ökologischen Kosten müssten dann erneut externalisiert werden, was Sturm and Vogt (2011) als einen klaren Beleg für das Versagen des Marktes ansehen würden.

Das entscheidende Problem besteht folglich darin, dass Produkte ausschließlich für die Technosphäre hergestellt werden (McDonough und Braungart 2013). So ist Kupfer in technologischen Systemen nahezu beliebig einsetzbar und hilfreich, in biologischen Systemen kann der Rohstoff jedoch extrem giftig sein. Ziel muss es daher sein, Produkte so zu gestalten, dass sie im Sinne eines Cradle-to-Cradle-Ansatzes auch in der Biosphäre und damit im Anschluss an die technische Nutzung einsetzbar sind. Hierzu bedarf es des Aufbaus eines verbindenden Nährstoffmanagements für die Techno- und Biosphäre. Menschliche Lebensweisen, die zu Grunde liegenden Prozesse und Produkte können so zum Nutzen für die Umwelt eingesetzt werden. Der Blick ist daher auf jene Innovationen zu richten, die das gegenwärtige Paradigma von Umweltschutz aufbrechen, indem sie Produkte und Prozesse realisieren, die eine nützliche stoffliche Verbindung zwischen Techno- und Biosphäre schaffen. Beispielhaft für diese Art des schöpferisch-zerstörerischen Prozesses steht das österreichische Unternehmen Gugler, das die weltweit erste Druckerei darstellt, die Druckerzeugnisse ohne schädliche Inhaltsstoffe und ausschließlich mit Substanzen herstellt, die wieder in den biologischen Kreislauf rückgeführt werden können (Gugler GmbH 2018). So werden die anfallenden Schlämme wieder der Biosphäre zugeführt, auch die Asche von verbrannten Druckprodukten kann bedenkenlos als Düngemittel wiederverwendet werden. Unter diesen Bedingungen ist es möglich, wirtschaftliche Aktivitäten mit einem hohen Durchsatz an Ressourcen ökologisch zu gestalten. Die prinzipielle Ablehnung eines grünen Kapitalismus übersieht derartige innovative Ansätze zur Bewältigung der Umweltkrise und ist damit kontraproduktiv.

\section{Rolle staatlicherPolitik}

\subsection{Gemanagte Energiewende}

Kallis (2011, S. 875) betont, dass deregulierte Märkte oft unkontrolliertes Chaos hervorgebracht haben und sich nicht für eine allmähliche Annäherung an eine nachhaltige Gesellschaft eignen, die zu unterscheiden weiß, welche Bereiche wachsen dürfen und welche nicht. Selbst Schumpeter spricht dem kapitalistischen Prozess die Fähigkeit zu einem kontinuierlichen Anpassungsprozess, wie es Meadows et al. (2006) für die Annäherung an die Tragfähigkeitsgrenze fordern, ab (Herzog und Honneth 2016, S. 57 ff.). Innovationen, die zur radikalen Veränderung etablierter wirtschaftlicher Kalkulationsgrundlagen führen, treten sprunghaft auf (Schumpeter 1994, S. 1119 f.) und sind für die Bewältigung der Umweltkrise dadurch zu unberechenbar. Wangler (2013, S. 213) erläutert hierzu, dass, bevor sich Innovationsprozesse gezielt auf die Bewältigung einer ganz bestimmten Art von Krise ausrichten können, eine Nachfrage nach etwaigen Gütern und Produkten bestehen und durch staatliche Politik weiter angekurbelt werden muss. Der Markt bedarf folglich eines institutionellen Rahmens, in dem es der Politik möglich ist, Innovationen zielgenau auszurichten. Solomon und Krishna (2011, S. 7427) sprechen hierbei von ",managed transitions".

Dass der Markt allein unzureichend ist, offenbaren die marktbasierten Ausschreibungsverfahren für erneuerbare Energien seitens der Bundesnetzagentur (Bundesnetzagentur 2018). Harriss-White und Harriss $(2007$, S. 81) betonen, dass die anvisierten Ausbauziele mit derlei Strategien ständig und weit unterschritten wurden. Doch wenn der Markt prinzipiell die Fähigkeiten zur Überwindung der Umweltkrise besitzt, warum setzt er sie dann nicht ein? Hierzu erläutert McCarthy (2015, S. 2486), das machtvolle Akteure des kapitalistischen Systems überhaupt nicht an einer Transformation des Energiesystems interessiert sind. Auch Stenzel und Frenzel (2008, S. 2645) sehen, dass die großen Energieversorger trotz grüner Rhetorik hartnäckig Widerstand leisten. Inwieweit dieser gebrochen werden kann, hängt nach Ćetkovic' und Buzogány (2016, S. 643) von den spezifischen nationalen politisch-ökonomischen Kontexten, die die Entwicklung regenerativer Energiesysteme beeinflussen, ab. Sie gehen dabei aber nicht von Kontexten jenseits der Marktwirtschaft aus, sondern sehen, indem sie verschiedene Formen des Kapitalismus vergleichen, in einer ganz bestimmten Ausprägung von Marktwirtschaft einen geeigneten Ansatz zur Krisenbewältigung. Kallis (2011, S. 875) hingegen betrachtet jegliche Form 
von Kapitalismus als einen institutionellen Verbund, dessen oberstes Ziel die Aufrechterhaltung von Wachstum und Akkumulation ist. McCarthy (2015, S. 2491) unterstützt diese These und behauptet, dass im Kontext der Umweltkrise jegliche kapitalistische Ordnung primär an der eigenen Rettung interessiert ist, nicht aber an der gesellschaftlichen Transformation in Richtung Nachhaltigkeit. Selbst den politischen Akteuren gehe es bei ihrem Engagement für Klimaschutz nur darum, die jeweils eigene Partei im politischen Wettbewerb neu zu positionieren, wie HarrissWhite und Harriss (2007, S. 83) behaupten.

Diese generelle Ablehnung jeglicher kapitalistischen Ordnung übergeht die bedeutsamen Unterschiede in der EnergiePolitikgestaltung, die wir im Folgenden kurz darlegen wollen.

Ćetkovic' und Buzogány (2016, S. 644 f.) zeigen auf, dass sich „Liberal Market Economies“ durch einen starken Wettbewerb auszeichnen. Sie orientieren sich an wirtschaftlichen Kriterien und bringen in unregelmäßigen Abständen bahnbrechende Innovationen hervor. Das bedeutet, dass zwar von radikalen Umstrukturierungen der Wirtschaft im Sinne Schumpeters (1912) auszugehen ist, Zeitpunkt und Richtung aber nicht steuerbar sind. In "Coordinative Market Economies“, so Ćetkovic' und Buzogány (2016) weiter, vollziehen sich Innovationsprozesse hingegen stufenweise und kontinuierlich. Der Fortschritt wird dabei von staatlicher Seite einer forschungsbasierten langfristigen Entwicklung der Wirtschaft untergeordnet. Beispielhaft hierfür steht die staatlich organisierte Transformation des Energiesystems in Brasilien in Folge der Ölpreisschocks. Zahlreiche Maßnahmen haben dazu beigetragen, die heimische Produktion und Konsumption von Bioethanol anzukurbeln. Die Regierung legte dabei großen Wert auf die Förderung von Forschung und Innovation in der Ethanolindustrie, was Brasilien den Weg zum Technologieführer im Bereich Ethanol ebnete (Solomon und Krishna 2011, S. 7424 f.). Auch in Deutschland achtete der Staat auf eine umfassende und stabile Unterstützung des Energiesystems (Stenzel und Frenzel 2008, S. 2649). So wurden technologiespezifische Einspeisetarife oberhalb des Marktpreises für Strom für einen Zeitraum von 20 Jahren garantiert (Kungl 2015). Darüber hinaus wurden zahlreiche Pilotprojekte zu erneuerbaren Energien gefördert, an denen Unternehmen sowie Forschungseinrichtungen beteiligt waren (Lauber und Jacobsson 2016). Diese stabilen Rahmenbedingungen erleichterten Investoren den Zugang zu Krediten und Darlehen. Der Aufbau des regenerativen Energiesystems wurde sogar von der regierungseigenen Bank gestützt (Ćetkovic' und Buzogány 2016, S.646f.). Wangler (2013, S. 212) ist davon überzeugt, dass aufgrund der hohen Produktionskosten für erneuerbare Energien sowie der monopolistischen Marktstrukturen konventioneller Energieversorgung eine Verbreitung grüner Technologien in Deutschland nur mit Hilfe staatlicher Intervention möglich war. Die politisch induzierte Stimulation der Nachfrage nach grünen Technologien und der damit einhergehende Effekt eines wachsenden Marktes konnte nach seinen Analysen die unternehmerischen Aktivitäten im regenerativen Sektor sowie die Innovationsrate signifikant erhöhen. Dies deckt sich mit der Feststellung von Solomon und Krishna (2011, S. 7429), dass die staatliche Unterstützung von Innovation auch indirekt über die Förderung und Erleichterung des Konsums von entsprechenden Produkten erfolgen kann. Harriss-White und Harriss (2007, S. 78 f.) sehen in den staatlichen Subventionen für den Verbrauch regenerativen

Stroms nur eine Kompensation für das Versagen des Marktes zur Implementierung regenerativer Energiesysteme. Ein gescheiterter Alleingang des Marktes muss jedoch nicht zwingend einen völligen Verzicht auf Wettbewerb nach sich ziehen, vielmehr sollten die Fähigkeiten des Marktes mittels staatlicher Kontrolle gezielt in den Aufbau der regenerativen Energieversorgung miteinbezogen werden. Beispielhaft hierfür steht England, wo die Politik lange Zeit eine liberale Haltung eingenommen und den Fokus auf einen technologieneutralen, kosteneffizienten Ausbau erneuerbarer Energien gelegt hatte. Die Folge war, dass der Ausbau allein den Marktmechanismen überlassen wurde und unausgereifte Technologien sowie kleinere Unternehmen keine Entwicklungschancen hatten (Stenzel und Frenzel 2008, S. 2648). Diese Form des Kapitalismus hemmte die Entwicklung eines heimischen Marktes für erneuerbare Energien. Kallis (2011, S. 875) sieht das Hemmnis dabei in den großen, machtvollen Unternehmen, die nicht an einer Abkehr von den für sie vorteilhaften, an Wachstum orientierten Verhältnisse interessiert sind. Die Etablierung eines nachhaltigen Gesellschaftssystems könne seiner Auffassung nach daher nicht unter den institutionellen Rahmenbedingungen des Kapitalismus erreicht werden. Tatsächlich ist es jedoch mit der Verknüpfung von Markt und Staat, d. h. mit der Etablierung ähnlicher institutioneller und politischer Rahmenbedingungen, wie es in der koordinierten Marktwirtschaft Deutschlands der Fall ist (z. B. staatlich garantierte Einspeisetarife, staatlich geförderte Energieprojekte, institutionelle Verdichtung, staatliche Green Investment Bank), gelungen, industrielles Wachstum und hohe Ausbauraten im regenerativen Energiesektor Englands zu erreichen (Ćetkovic' und Buzogány 2016, S. 648 f.).

Jänicke (2007, S. 131) sieht in dem Transfer erfolgreicher politischer Regulierungen eine wesentliche Voraussetzung für Innovation, ganz im Sinne eines regulativen Kapitalismus. Ćetkovic' und Buzogány (2016, S. 652 f.) verweisen jedoch auf die Grenzen der Übertragbarkeit politisch-institutioneller Rahmenbedingungen. Harriss-White und Harriss (2007, S. 78 ff.) 
bemerken, dass der Aufbau regenerativer Energiesysteme in England zu lange in den Zuständigkeitsbereichen vieler, teils zerstrittener Ministerien gelegen hatte und unzureichend koordiniert wurde. In diese Lücke konnten Interessenvertreter der Industrie vorstoßen und so einen erheblichen Einfluss auf die Politikgestaltung im Energiebereich ausüben, was zu einer Verstrickung der Energiepolitik mit den Interessen des Kapitals geführt hat. Genau diese Art der politischen Verstrickung war bei der Umstellung des französischen Energiesystems von Öl auf Kernenergie wiederum ein entscheidender Erfolgsfaktor (Solomon und Krishna 2011, S. 7425 f.). Doch unabhängig davon sind die Unterschiede zwischen dem englischen und dem deutschen regenerativen Energiesystem v. a. auf spezifische nationale politischökonomische Kontexte zurückzu-führen. So ist der Vorsprung der deutschen Energiewende auch das Ergebnis einer wesentlich stärkeren industriellen Basis, die Deutschland die Gelegenheit gab, seine große Ingenieursund Maschinenbautradition im neuen Energiesektor fortzuführen (Ćetkovic' und Buzogány 2016, S.647). Erfolgsfaktoren sind zudem die stabilere staatliche Unterstützung der Industrie, eine auf den grünen Energiesektor ausgerichtete Ausbildung sowie die institutionelle Einbettung der Aushandlungsprozesse in regionale und lokale Strukturen. Die Gestaltung der Energiewende über Bottom-upProzesse hat nicht zuletzt zu einer großen Akzeptanz geführt (Gailing et al. 2013) und industrielles Wachstum und Innovationen dadurch kontinuierlich hervorbringen können. Nach den nationalen Fallbeispielen wollen wir darlegen, welche Rahmenbedingungen für einen erfolgreichen grünen Kapitalismus im Allgemeinen gegeben sein müssen: Für Meadows et al. (2006) müsste die Bevölkerung besser im Umweltbereich ausgebildet, das finanzielle Risiko zur Entwicklung neuer Technologien staatlich abgefangen und Institutionen zur Entwicklung sowie Verbreitung neuer Technologien geschaffen werden. Des Weiteren bedürfe es der organisatorischen Fähigkeiten von Staat und Wirtschaft, sich auf die entscheidenden Herausforderungen der Gesellschaft zu konzentrieren. Solomon und Krishna (2011, S. 7427) erläutern, dass zur Transformation von Energiesystemen drei Voraussetzungen gegeben sein müssen. Dazu zählen zum einen Basisinnovationen aus Nischenmärkten heraus (vgl. Geels 2011; Raven et al. 2016), die durch die staatlichen Organe geschützt und gefördert werden. Des Weiteren bedarf es eines spezifischen soziotechnischen Regimes, mit einem Netzwerk an Akteuren und sozialen Gruppen, innerhalb dessen koordinierte Verhaltensweisen und ein gemeinsames Wertesystem aufgebaut werden. Schließlich ist entscheidend, in welchen makroökonomischen Kontext Innovationen sowie Netzwerke eingebettet werden und von welchen kulturellen sowie politischen Wurzeln sie gespeist werden. Im Sinne eines „Energy Keynesianism“, bei dem die Produktionsprozesse in der Energiewirtschaft über die gesamtgesellschaftliche Nachfrage gesteuert und so regenerative Energiesysteme stabil aufgebaut werden, sieht McCarthy (2015, S. 2498) beim Staat ein großes Gestaltungspotenzial. Zentrale staatliche Handlungsfelder liegen dabei in der Erschließung nationaler Energiepotenziale, im Abbau von Investitionshürden und in der internationalen Zusammenarbeit im Kontext von Energiepolitik. Ähnlich äußern sich auch Ćetkovic' und Buzogány (2016, S. 644), für die eine zielorientierte staatliche Energiepolitik zum einen den Aufbau eines heimischen Marktes zur Erschließung des regenerativen Energiepotenzials umfasst, zum anderen aber auch industriepolitische Maßnahmen einleitet, die die Innovationskraft der regenerativen Energiebranche fördern, Arbeitsplätze schaffen und die internationale Wettbewerbsfähigkeit der Industrie stärken. Die Akzeptanz regenerativer Energiesysteme hänge dabei von der Fähigkeit es Staates ab, gesellschaftliche Partizipation in Form von Mitsprache und monetärer Teilhabe zu gewährleisten, wie DevineWright (2005), Aitken (2010) sowie Walter und Gutscher (2013) bereits veranschaulicht haben. Doch auch Forschung und Entwicklung müssen dazu bereit sein, mit den staatlichen Bemühungen über einen größeren Zeitraum hinweg Hand in Hand zu gehen (Solomon und Krishna 2011, S. 7429). Förderlich ist es nach Ansicht der Autoren zudem, den Ausbau eines neuen Energiesystems mit dem Nationalstolz zu verknüpften. Dies sei in Frankreich der Fall gewesen, wo der starke Ausbau von Atomkraftwerken den Franzosen die Energieunabhängigkeit zurückgeben konnte. In diesem Zusammenhang hat sich gezeigt, dass die Zentralisierung der Energieversorgung sehr wirksam sein kann. McCarthy (2015, S. 2485) erläutert diesbezüglich, dass gerade die Koordinierung vieler dezentraler Energiesysteme einer zentralen energiewirtschaftlichen Steuerung bedarf. Die Zentralisierung der Energieversorgung bietet nicht zuletzt große Vorteile: So konnten Bauknecht et al. (2015, S. 11 ff.) darlegen, dass die durchschnittlichen Kosten einer erzeugten Einheit Elektrizität (Gestehungskosten) bei zentralen Energiesystemen wesentlich geringer sind, als dies bei dezentralen Systemen der Fall ist. Zudem führe der vergleichsweise geringe Ertrag individueller Anlagen eines dezentralen Systems zu einem größeren Gesamtbedarf an Anlagen, wodurch auch die Kraftwerksinvestitionen deutlich höher ausfallen (Gesamtinvestitionen). Darüber hinaus betonen die Forscher, dass im Kontext des Aufbaus dezentraler Strukturen die Energienetze ungleich stärker angepasst werden müssten. Während die Kraftwerke des zentralen Systems überwiegend auf der Übertragungsnetzebene errichtet werden und diese Netzebene relativ gut ausgebaut ist, speisen dezentrale Anlagen verstärkt in die unzureichend ausgebaute Verteilnetzebene ein, die kaum in der Lage ist, die anfallenden Strommengen aufzunehmen (Netzkosten). Schließlich sind dezentrale Systeme auch aus ökologischer Sicht als kritisch zu betrachten, da die energetische Effizienz 
aufgrund der verstärkten Nutzung von Flexibilitätsoptionen (z. B. Energiespeicher, Lastmanagement) und den damit einhergehenden Umwandlungs- sowie Wirkungsgradverlusten stark herabgesetzt wird (Ökologische Kosten).

Zusammenfassend kann festgehalten werden, dass es mit Blick auf die Lösung der Umweltkrise von Vorteil ist, Märkte mittels staatlicher Maßnahmen zu begleiten. Die Energiewende in Deutschland ist hierfür ein Beispiel, hat der Staat über die Einführung des Erneuerbare-Energien-Gesetz doch wesentlich zur Erhöhung der Innovationsrate im regenerativen Energiesektor beigetragen (Kungl 2015). Nicht zuletzt weist Deutschland innerhalb der Europäischen Union die meisten technologischen Patente im Solar-und Windenergiebereich auf (Ćetkovic' und Buzogány 2016, S. 647). Doch trotz dieses Erfolgs wird das deutsche Energiesystem gegenwärtig wieder stärker marktwirtschaftlich ausgerichtet (BMJV 2017). Diese Kehrtwende ist darauf zurückzuführen, dass die ökonomische Effizienz von Einspeisetarifen als zu gering eingeschätzt wird (Frondel et al. 2013). Daraus erwächst die Gefahr, dass die Anreize für den Ausbau erneuerbarer Energien wieder schwächer werden. Selbst private Investoren im Bereich erneuerbarer Energien haben aus Gründen der Investitionssicherheit ein großes Interesse daran, dass die politischen Rahmenbedingungen nicht gänzlich verschwinden (Bürer und Wüstenhagen 2009, S. 4997). Ćetkovic' und Buzogány (2016, S. 653) empfehlen daher, den Fokus nicht allein auf Kosteneffizienz und kurzfristige Entwicklungen zu richten, sondern jenseits des Marktes politische und institutionelle Rahmenbedingungen zu schaffen, die industrielles Wachstum und Innovationen hervorbringen, breite bürgerliche Partizipation erlauben und die spezifischen komparativen Kostenvorteile der einzelnen Länder entfalten helfen.

\subsection{BerücksichtigungsozialerFaktoren}

In einem letzten Schritt wird die These, dass der Aufbau regenerativer Energiesysteme einen Beitrag zur Überwindung sozialer Ungleichheiten im Rahmen der Energieversorgung leistet, überprüft.

Der Stand der Forschung hierzu offenbart, dass Umweltschutz und Wirtschaftswachstum auf Basis von effizienten Technologien, Wettbewerb sowie staatlichen Interventionen nur selten ohne soziale Nebenwirkungen Hand in Hand gehen. Eine Energiewende verfehlt aber nach Ansicht von Bradley und Hedrén (2014) ihr Ziel, wenn es nur darum geht, das Energiesystem zu modernisieren, ohne dabei jedoch auch die sozialen Strukturen, die dahinterstehen, zu transformieren. Dies betrifft nicht nur die europäischen Transformationsprozesse, sondern die Energiewenden weltweit. Speziell bei Energiegroßprojekten im Globalen Süden trägt der Ausbau erneuerbarer Energien immer wieder zur Verfestigung bereits bestehender, weit zurückreichender gesellschaftlicher Ungleichheiten bei. So konnten Yenneti et al. (2016) aufzeigen, dass der Charanka Solar Park im indischen Gujarat auf Flächen errichtet wurde, die der ärmeren lokalen Bevölkerung seit mehreren Jahrzehnten als Lebensgrundlage dienten. Der verwehrte Zugang zu diesen Flächen und die sukzessive Enteignung von ohnehin schon unterprivilegierten Bevölkerungsschichten seitens des Staates sind damit eine direkte Folge der ökologischen Modernisierung der Wirtschaft (Levien 2013). Baka (2013) spricht hierbei von „energy dispossessions“, wobei dieses Phänomen auch bei groß angelegten Windparks und weltweit festgestellt wurde (Avila 2018; Cowell 2010).

Harvey (1996) hat darauf hingewiesen, dass speziell arbiträre Raumkategorisierungen oftmals auf einer marktwirtschaftlich-kapitalistischen Logik basieren. Dabei üben wenige machtvolle Akteure die Kontrolle über lokalisierte Ressourcen (z. B. Flächen) aus. Cowell (2010) wittert diesbezüglich beispielhaft und stellvertretend im Aufbau britischer regenerativer Energiesysteme eine Marginalisierung vorbelasteter, industrialisierter Räume, die aufgrund einer konstruierten Hierarchie von Landschaften als profane Regionen das Gros des Ausbaus erneuerbarer Energien zu tragen haben. Dabei ist überhaupt nicht geklärt, wann es sich um schützenswerte Räume handelt und welche energiebezogenen Flächennutzungen hierdurch auszuschließen sind (Palmas et al. 2015). Vordergrün dig erweckt der Einsatz ausgeklügelter Geographischer Informationssysteme (GIS) zur Ausbau-Planung erneuerbarer Energien den Eindruck, dass soziale Parameter exakt erfasst werden. Tatsächlich ist die soziale Tiefe von Planung durch die politischen Rahmenbedingungen innerhalb Europas klar gedeckelt, mit dem Ziel, der Politik eine räumliche Steuerung aus der Distanz zu erlauben. Durch diese Generalisierung wird räumliche Planung sozial erst kalkulierbar (Murdoch 2000). Liljenfeldt (2015) hat diesbezüglich herausgefunden, dass in Finnland, Norwegen und Schweden die Eingriffsmöglichkeiten räumlicher Planung stark zu Gunsten effizienter Top-down-Verfahren eingeschränkt wurden, um dadurch die Ziele der Energiewende schneller erreichen zu können. Diese Art der räumlich-technischen Planung, die die lokalen Kontexte entsprechend den politischen Vorgaben ignorieren oder einbinden kann, ist die Machtbasis übergeordneter Energieplanung (Demeritt 2001). Die dabei entstehenden Planungskarten, die die Ziele von Energiepolitik in räumlich-administrative Formen gießen und die Heterogenität der Teilräume auf kontrollierbare Kategorien reduzieren, sind dabei der visuelle Ausdruck dieser Macht. Cowell (2010) spricht hierbei vom Phänomen der „foreshadowed search areas“. Die Sozialwissenschaften werden hierzu oftmals instrumentalisiert, indem sie helfen sollen, soziale Blockaden für energiepolitische Ambitionen zu lösen (Shove 
1998). Das Soziale wird dadurch je nach Interessenlage manipuliert (Bulkeley et al. 2005) und die lokalen Kontexte von den nationalen Zielen abgekoppelt (Cowell 2010). Reziprozität zwischen übergeordneter Energiepolitik und lokalen Kontexten ist jedoch wichtig, da die Opposition zu erneuerbaren Energien meistens aus vor Ort lebenden Menschen hervorgeht (Van d. Horst 2007). Zoellner et al. (2008) kritisieren daher, dass sich die Energieforschung zu lange einseitig auf die technischen Aspekte der Energiewende konzentriert hat und soziale Themen so aus dem Blickfeld geraten konnten. Dies wirkte sich auf die Planungspraxis aus und brachte vielerorts lokale Proteste hervor (Aitken 2010). Gerade die mangelhafte akademische Reflexion quantitativer Vorgaben zum Ausbau erneuerbarer Energien konnte den Blick auf die tieferen Beweggründe lokaler Opposition verstellen. Wissenschaftliche Problemstellung und Zielsetzung orientieren sich nur mehr an der Frage, wie die quantitativen Vorgaben der Energiepolitik trotz einer verringerten Akzeptanz umsetzbar sind. Dies bezeichnen Ellis et al. (2007) als ideologisch aufgeladene Pro-Energie-Haltung, die alternative Sichtweisen ignoriert. Selbst das Recht auf Mitbestimmung muss als problematisch angesehen werden, wenn es allein dem rein kosmetischen Zweck dient, bereits getroffene Entscheidungen zu legitimieren (Hildyard et al. 2001). Devine-Wright (2005) wirbt deshalb für die planerische Berücksichtigung sozialer Distanzen, die als ein zentrales Maß für Gerechtigkeit angesehen werden können. Je gerechter ein Planungsprozess wahrgenommen wird, desto größer ist nicht zuletzt die Akzeptanz (Zoellner et al. 2008). Walter and Gutscher (2013) bemerken wiederum, dass gerechtes Empfinden und lokale Akzeptanz in engem Bezug zu den von der Bevölkerung assoziierten Kosten für Mensch und Umwelt stehen. Die landschaftlichen Kosten, die durch die Technisierung ländlicher Räume entstehen, sind dabei sicherlich von großer Bedeutung (Nadaï und Van d. Horst 2010). Wenn diese Kosten künftig berücksichtigt werden, darf dabei aber nicht mehr die Tatsache übergangen werden, dass Landschaft für alle Menschen in allen Regionen eine kulturelle Bedeutung besitzt, unabhängig davon, ob diese unattraktiv, gewöhnlich oder schön ist (Blaschke et al. 2013). Nur durch diese Planungsperspektive wird es möglich sein, den Aufbau regenerativer Energiesysteme langsfristig sozial ausgewogen gestalten zu können.

Neben dieser fundamentalen Kritik ist jedoch festzuhalten, dass die Verhinderung zahlreicher Energieprojekte gerade in den westlichen Marktwirtschaften auch das Ergebnis einer großen demokratischen Vitalität ist, die zur Grundlage zahlreicher ergebnisoffener Aushandlungsprozesse werden und dabei sämtlichen gesellschaftlichen Interessen Rechnung tragen konnte (Naturschutz, Denkmalschutz, Heimatpflege, Industrie etc.) (Walter und Gutscher 2013; Raven et al. 2016). In sozialistischplanwirtschftlichen Wirtschaftssystemen ist diese Vitalität nicht annähernd gegeben und teilweise sogar unzulässig (Andrews-Speed 2012). Die marktwirtschaftlichen Energiewenden haben damit ein großes Potenzial für bürgerliches Engagement eröffnet (Gailing et al. 2013), so dass die Energiekonsumenten nicht mehr einseitig in die Abhängigkeit großer Energiekonzerne geraten, sondern ihrerseits zu Energieproduzenten werden und am Aufbau regenerativer Energiesysteme beteiligt sind (Van d. Schoor und Scholtens 2015).

Letztlich ist zu konstatieren, dass mit marktbasierten Energiewenden prinzipiell das Potenzial einer größeren sozialen Ausgewogenheit im Rahmen der Energieversorgung einhergeht, dieses jedoch unzureichend ausgeschöpft und der Verstärkung sozialer Ungleichheiten dadurch der Weg bereitet wird.

\section{Fazit}

In diesem Aufsatz argumentierten wir, dass der Kapitalismus nicht nur wesentlich robuster ist, als von seinen Kritikern angenommen, sondern darüber hinaus vielversprechende Fähigkeiten im Hinblick auf die Überwindung der Umweltkrise anbietet.

Zu Beginn des Aufsatzes haben wir die Beziehung zwischen Kapitalismus sowie fossilen Energieträgern beschrieben und dargelegt, welche dramatischen ökologischen Folgen daraus erwachsen. Es wurde deutlich, dass viele Wissenschaftler in dieser Beziehung eine Unauflöslichkeit sehen und davon ausgehen, dass, wenn die Zeit fossiler Energieträger endet, das Ende des Kapitalismus zwangsläufig folgen wird. Dementgegen konnte aufgezeigt werden, dass der Kapitalismus bei einem Ende fossiler Energieträger fortbestehen und seine Produktivität auch im Rahmen eines regenerativen Energiesystems beibehalten kann. Innovative Speicherkonzepte, Gleichstromübertragung und Smart Grids spielen dabei eine wesentliche Rolle. Dementsprechend muss die Behauptung, dass ein regeneratives Energiesystem in jedem Fall andere gesellschaftliche Rahmenbedingungen benötigt, als jene, die das fossile Energiesystem hervorgebracht hätte, verworfen werden. Des Weiteren haben wir darlegen können, dass die Krise ein wesentliches Element kapitalistischer Ordnung ist und ein krisenhafter Zustand sogar die notwendigen Voraussetzungen zur Transformation der Wirtschaft in Richtung Nachhaltigkeit schaffen kann. Innovationen spielen dabei eine zentrale Rolle. Dabei wurde argumentiert, dass gerade der Konkurrenzkapitalismus basale Innovationen hervorzubringen vermag. Den entscheidenden sozialen Vorteil eines wettbewerbsorientieren kapitalistischen Systems sehen wir folglich darin, dass, entsprechend Schumpeters Prinzip der 
„schöpferischen Zerstörung", ein maximaler Anreiz für unternehmerische Initiativen besteht. Nach der "Theorie der wirtschaftlichen Entwicklung" kann dieser Anreiz in den von Harris (2013) bevorzugten, aber nicht näher erläuterten "socialist markets" nicht gegeben sein. Dieser Anreiz ist jedoch von zentraler Bedeutung, geht er doch unmittelbar mit einer größeren Wahrscheinlichkeit bahnbrechender Innovationen sowie einer generell größeren Innovationskraft in den Bereichen Technologie, Bildung, Ausbildung, Forschung, soziale Infrastruktur, Medizin, Umwelt etc. einher (Schumpeter 1994; Iversen 2005; Wangler 2013).

Die vielversprechenden Möglichkeiten eines grünen Kapitalismus wurden ausführlich dargelegt und stets im Kontext des Konkurrenzkapitalismus betrachtet. Die vorherrschende Kritik am Kapitalismus geht aber nur noch selten von diesem Kontext aus und vernachlässigt dadurch die Gestaltung von Rahmenbedingungen, aus denen Unternehmer mit großer Innovationskraft emporsteigen können. Dabei setzt die Kritik an einer sehr viel späteren Entwicklungsstufe des wirtschaftlichen Systems an, bei der sich sozioökonomische Ungerechtigkeiten als Folge ökonomischer, aber auch anderer Faktoren stark und in großer Komplexität manifestiert haben. Die reine Kapitalismuskritik erscheint dabei als ein zu oberflächliches Erklärungsmodell, das mit Argumenten agiert, die gerade jene fruchtbaren Ansätze zur Überwindung der Energie- und Umweltkrise übersehen, die gegenwärtig aus den zahlreichen Vorgängen schöpferischer Zerstörung erwachsen. Der beschriebene Cradleto-Cradle-Ansatz ist nur ein Beispiel für ein Konzept, das über marktbasierte Innovationen Altes verwirft und Neues etabliert. Davon abgesehen konnte aber auch aufgezeigt werden, dass Wettbewerb und Märkte allein nicht ausreichen werden, um die globale Umweltkrise gezielt lösen zu können. Hier bedarf es der politischen Initiative, die es durch den Aufbau entsprechender institutioneller Rahmenbedingungen schafft, die gesellschaftlichen Kräfte im Hinblick auf die ökologischen Fragen unserer Zeit zu bündeln und Innovationen so gezielt fördert. DieSinnhaftigkeit von staatlichen Maßnahmen hängt dabei immer auch von den jeweils nationalen politischökonomischen Kontexten ab. Eine generelle Ablehnung kapitalistischer Ordnungen übergeht diese Vielfalt an Kontexten und läuft daher Gefahr, bedeutende Determinanten der Krisenbewältigung zu übersehen. Dem Staat fällt dabei eine zentrale Rolle zu, denn er muss die Handlungen der entscheidenden Akteure in geeignete institutionelle Strukturen einbetten. Diesbezüglich stellten Ćetkovic' und Buzogány (2016, S. 649) fest, dass in liberaleren Ausprägungen des Kapitalismus der Mangel an politisch-institutionellen Instrumenten die notwendige Abstimmung von Aktivitäten zwischen Staat, Industrie und Finanzsektor hemmt. Solomon und Krishna (2011, S. 7427) konnten darlegen, dass durch diesen Mangel die anvisierte Transformation des Energiesystems in den USA nach der Ölkrise nicht gelungen ist, da unter anderem keine geeigneten Rahmenbedingungen für Innovationen in Nischenmärkten geschaffen wurden. Schließlich muss der Staat auch dafür sorgen, dass die sozialen Dimensionen nicht aus dem Blickfeld von Industrie und Planern geraten, da die Akzeptanz eine grundlegende Voraussetzung für einen erfolgreichen Aufbau regenerativer Energiesysteme bildet. Dass dies speziell in der räumlichen Planung erneuerbarer Energien noch nicht hinreichend berücksichtigt wurde und zu Teils starken sozialen Verwerfungen geführt hat, konnte beispielhaft aufgezeigt werden.

Zusammenfassend kann festgehalten werden, dass, wenngleich die fossilen Energieträger und der auf ihnen gründende Kapitalismus in die Umweltkrise geführt haben, die Überwindung der Krise nicht zwangsläufig durch die Überwindung marktwirtschaftlicher Ansätze erfolgen muss, sondern staatlich begleitete, wettbewerbsorientierte Marktwirtschaften auf Basis regenerativer Energiesysteme eine große Wirksamkeit im Hinblick auf Nachhaltigkeit entfalten können.

\section{Literatur}

Aitken M (2010) Why we still don't understand the social aspects of wind power: A critique of key assumptions within the literature. Energy Policy 38(4):1834-1841

Alcott B (2005) Jevons' Paradox. Ecol Econ 54(1):9-21

Altvater E (2005) Das Ende des Kapitalismus wie wir ihn kennen. Eine radikale Kapitalismuskritik. Westfälisches Dampfboot, Münster

Altvater E (2007) The social and natural environment of fossil capitalism. Social Regist 43:37-58

Anagnostopoulos JS, Papantonis DE (2008) Simulation and size op- timization of a pumped-storage power plant for the recovery of wind-farms rejected energy. Renew Energy 33:1685-1694

Andrews-Speed P (2012) The governance of energy in China -transition to a low-carbon economy. Palgrave Macmillan, New York Arentsen M, Bellekom S (2014) Power to the people: local energy initiatives as seedbeds of innovation? Energy Sustain Soc 4(2):1-12 Avila S (2018) Environmental justice and the expanding geography of wind power conflicts. Sustain Sci 13(3):599-616 
Baccini P, Brunner PH (2012) Metabolism of the Anthroposphere: analysis, evaluation, design. MIT Press, Cambridge

Baka J (2013) The political construction of wasteland: governmentality, land acquisition and social inequality in South India. Dev Change 44(2):409-428

Bardi U (2009) Peak oil: The four stages of a new idea. Energy 34(3):323-326

Bauknecht D, Vogel M, Funcke M (2015) Energiewende - Zentral oder dezentral? Diskussionspapier im Rahmen der Wissenschaft- lichen Koordination des BMBF Förderprogramms: „Umwelt- und Gesellschaftsverträgliche Transformation des Energiesystems“. https://www.oeko.de/oekodoc/2368/2015-534-de.pdf. Zugegrif- fen: 23. Nov. 2018

Benson SM, Orr FM (2008) Carbon dioxide capture and storage. Mater Res Soc Bull 33:303-305

Bettini G, Karaliotas L (2013) Exploring the limits of peak oil: naturali- sing the political, de-politicising energy. Geogr J 179(4):331-341 Blaschke T, Biberacher M, Gadocha S, Schardinger I (2013) “Ener- gy landscapes": Meeting energy demands and human aspirations.

Biomass Bioenergy 55:3-16

Böhm S, Misoczky MC, Moog S (2012) Greening capitalism? A mar- xist critique of carbon markets. Organ Stud 33(11):1617-1638

Bradley K, Hedrén J (2014) Utopian thought in the making of green futures. In: Bradley K, Hedrén J (Hrsg) Green Utopianism: perspec- tives, politics and micro-practices. Routledge, New York, Oxon, S 1-20

Brand U (2016) Green economy, green capitalism and the imperial mode of living: limits to a prominent strategy, contours of a possible new capitalist formation. Fudan J Humanit Soc Sci 9(1):107-121 Bridge G (2010) Geographies of peak oil: The other carbon problem.

Geoforum 41(4):523-530

Bridge G, Bouzarovski S, Bradshaw M, Eyre N (2013) Geographies of energy transition: Space, place and the low-carbon economy. Energy Policy 53:331-340

Buch-Hansen H (2018) The prerequisites for a Degrowth paradigm shift: insights from critical political economy. Ecol Econ 146:157-163

Bulkeley H, Watson M, Hudson R, Weaver P (2005) Governing municipal waste: towards a new analytical framework. J Environ Policy Plan 7(1):1-24

Bundesministerium der Justiz und für Verbraucherschutz (BMJV) (2017) Gesetz für den Ausbau erneuerbarer Energien. http:// www.gesetze-im-internet.de/eeg_2014/. Zugegriffen: 8. März 2018

Bundesnetzagentur (2018) Leitungsvorhaben. https://www.netzausbau. de/leitungsvorhaben/de.html. Zugegriffen: 25. April 2019

Bürer MJ, Wüstenhagen R (2009) Which renewable energy policy is a venture capitalist's best friend? Empirical evidence from a survey of international cleantech investors. Energy Policy 37(12):4997-5006

Castree N (2010) Crisis, continuity and change: Neoliberalism, the left and the future of capitalism. Antipode 41(S1):185-213

Ćetković S, Buzogány A (2016) Varieties of capitalism and clean ener-gy transitions in the European Union: When renewable energy hits different economic logics. Clim Policy 16(5):642-657

Chisholm M (1990) The increasing separation of production and consumption. In: Turner BL, Clark WC, Kates RW, Richards JF, Ma- thews JT, Meyer WB (Hrsg) The earth as transformed by human action. Global and regional changes in the biosphere over the past 300 years. Cambridge University Press, Cambridge, S87-102

Clausius R (1850) Ueber die bewegende Kraft der Wärme und die Gesetze, welche sich daraus für die Wärmelehre selbst ableiten las- sen. Poggendorffs Ann 79:368-397

Cowell R (2010) Wind power, landscape and strategic, spatial planning-The construction of "acceptable locations" in Wales. Land use policy 27:222-232

Daly HE (1991) Steady-state economics, 2. Aufl. Island Press, Washington DC

Daly HE (1995) On Nicholas Georgescu-Roegen's contributions to economics: An obituary essay. Ecol Econ 13:149-154

Daly HE (2005) Economics in a full world. Sci Am 293:100-107 Demeritt D (2001) Scientific forest conservation and the statistical picturing of nature's limits in the Progressive era United States. Soc Space 19:431-459

Devine-Wright P (2005) Beyond NIMBYism: towards and integrated framework for understanding public perceptions of wind energy. Wind Energy 8:125-139

Duthel H (2013) Politische Ökonomie. Books on Demand, Norderstedt

Ebeling W, Freund J, Schweitzer F (1998) Komplexe Strukturen: Entropie und Information. BG Teubner, Leipzig

Ellis G, Barry J, Robinson C (2007) Many ways to say "no", different ways to say "yes": applying Q-methodology to understand public acceptance of wind farm proposals. J Environ Plan Manag 50(4):517-551

Embshoff D, Giegold S (2008) Solidarische Ökonomie im globalisierten Kapitalismus. VSA, Hamburg 
European Commission (EC) (2018) 2050 long-term strategy. htt- ps://ec.europa.eu/clima/policies/strategies/2050_bg.

Zugegriffen:

25. April 2019

Fares R (2015) Renewable energy intermittency explained: challen- ges, solutions, and opportunities. https://blogs.scientificamerican. com/plugged-in/renewable-energy-intermittency-explainedchallenges-solutions-and-opportunities/. Zugegriffen: 8. Febr. 2018

Frondel M, Schmidt CM, Aus dem Moore N (2013) Marktwirtschaftliche Energiewende: Ein Wettbewerbsrahmen für die Strom- versorgung mit alternativen Technologien. Z Energiewirtsch 37(1):27-41

Gailing L, Hüesker F, Kern K, Röhring A (2013) Die räumliche Gestaltung der Energiewende zwischen Zentralität und Dezentra- lität. Explorative Anwendung einer Forschungsheuristik. https:// leibniz-irs.de/fileadmin/user_upload/IRS_Working_Paper/wp_ energiewende_raum_zentral_dezentral.pdf. Zugegriffen:22. Apr. 2018

García-Olivares A, Solé J (2015) End of growth and the structural instability of capitalism-From capitalism to a Symbiotic Econo- my. Futures 68:31-43

Gawel E, Strunz S, Lehmann P (2012) The German Energiewende under attack: Is there an irrational Sonderweg? https://www. econstor.eu/bitstream/10419/64555/1/726590568.pdf. Zugegrif- fen: 23. Mai 2018

Geels FW (2011) The multi-level perspective on sustainability transitions: Responses to seven criticisms. Environ Innov Soc Transit 1(1):24-40

Georgescu-Roegen N (1971) The entropy law and the economic process. Havard University Press, Harvard

Georgescu-Roegen N (1986) The entropy law and the economic pro- cess in retrospect. East Econ J 7(1):3-25

German Aerospace Center (DLR) (2007) Concentrating Solar Power for Seawater Desalination. http://www.dlr.de/tt/Portaldata/41/

Resources/dokumente/institut/system/projects/aqua-csp/AQUA- CSP-Full-Report-Final.pdf. Zugegriffen: 8. Juni 2018

German Aerospace Center (DLR) (2006) Trans-mediterranean interconnection for concentrating solar power. https://www.dlr.de/ tt/Portaldata/41/Resources/dokumente/institut/system/projects/ WP00_TRANS-CSP-Introduction_and_SummaryFinal.pdf. Zu- gegriffen: 25. April 2019

Grossmann WD, Grossmann I, Steininger KW (2014) Solar electricity generation across large geographic areas. Part II: A Pan-Amer- ican energy system based on solar. Renew Sustain Energy Rev 32:983-993

Gugler GmbH (2018) Cradle to Cradle Druckprodukte: Innovation aus Österreich. https://www.gugler.at/print/nachhaltigkeit/cradle-to- cradletm-druckprodukte-innovation-ausoesterreich.html. Zuge- griffen: 25. Sept. 2018

Hadjipaschalis I, Poullikkas A, Efthimiou V (2009) Overview of current and future energy storage technologies for electric power ap- plications. Renew Sustain Energy Rev 13:1513-1522

Harris J (2010) Going green to stay in the black: transnational capitalism and renewable energy. Race $\mathrm{Cl}$ 52(2):62-78

Harris J (2013) Can green capitalism build a sustainable society? Int Crit Thought 3(4):468-479

Harriss-White B, Harriss E (2007) Unsustainable capitalism: the politics of renewable energy in the UK. Soc Regist 43:72-101 Harvey D (1996) Justice, nature and the geography of difference. Blackwell Publishers Inc, Malden

Harvey D (2011) Roepke lecture in economic geography-crises, geo-graphic disruptions and the uneven development of political re- sponses. Econ Geogr 8:1-22

Herzog L, Honneth A (Hrsg) (2016) Joseph A. Schumpeter - Schriften zur OOkonomie und Soziologie. Suhrkamp, Berlin

Hildyard N, Hegde P, Wolvekamp P, Reddy S (2001) Pluralism, parti- cipation and power: joint forest management in India. In: Cooke B, Kothari U (Hrsg) Participation: the new tyranny? Zed Books, London, New York, S 56-71

Huber MT (2009) Energizing historical materialism: Fossil fuels, space and the capitalist mode of production. Geoforum 40(1):105-115 Huber MT (2013) Fueling capitalism: oil, the regulation approach, and the ecology of capital. Econ Geogr 89(2):171-194

Iversen T (2005) Capitalism, democracy, and welfare. Cambridge Uni- versity Press, Cambridge

Jänicke M (2007) Trendsetter im „regulativen Kapitalismus“: Das Beispiel umweltpolitischer Pionierländer. In: Holzinger K, Jörgens H, Knill C (Hrsg) Transfer, Diffusion und Konvergenz von Politi- ken. VS, Wiesbaden, S 131-149

Jensen SH, Larsen PH, Mogensen M (2007) Hydrogen and synthetic fuel production from renewable energy sources. Int J Hydrogen Energy 32(15):3253-3257

Kaberger T, Mansson B (2001) Entropy and economic processes-physics perspectives. Ecol Econ 36(1):165-179 
Kallis G (2011) In defence of degrowth. Ecol Econ 70(5):873-880

Kallis G, Martinez-Alier J, Norgaard RB (2009) Paper assets, real debts: an ecological-economic exploration of the global econo- mic crisis. Crit Perspect Int Bus 5(1-2):14-25

Kenis A, Lievens M (2016) Greening the economy or economizing the green project? When environmental concerns are turned into a means to save the market. Rev Radic Polit Econ 48(2):217-234

Kinder J (2016) The coming transition: fossil capital and our energy future. Social Democr 30(2):8-27

Kranert M, Cord-Landwehr K (2010) Einführung in die Abfallwirt- schaft. Springer, Heidelberg

Kungl G (2015) Stewards or sticklers for change? Incumbent energy providers and the politics of the german energy transition. Energy Res Soc Sci 8:13-23

Lauber V, Jacobsson S (2016) The politics and economics of constructing, contesting and restricting socio-political space for renewa- bles-the German renewable energy act. Environ Innov Soc Tran- sit 18:147-163

Levien M (2013) Regimes of dispossession: from steel towns to special economic zones. Dev Change 44(2):381-407

Liljenfeldt J (2015) Legitimacy and efficiency in planning proces- ses-(how) does wind power change the situation? Eur Plan Stud 23(4):811-827

Marques P (2008) Localised technological change: towards the economics of complexity. J Econ Geogr 9(2):288-290

Martinez-Alier J (1987) Ecological economics: Energy environment and society. Blackwell Publishers Inc, Oxford

Martinez-Alier J (2012) Environmental justice and economic degrowth: an alliance between two movements. Capitalism Nat Social 23(1):51-73

Matek B, Gawell K (2015) The benefits of Baseload Renewables: a misunderstood energy technology. Electr J 28(2):101-112 Mathews J (2011) Naturalizing capitalism: The next Great

Transformation. Futures 43(8):868-879

McCarthy J (2015) A socioecological fix to capitalist crisis and climate change? The possibilities and limits of renewable energy. Environ Plan A47:2485-2502

McDonough W, Braungart M (2013) The upcycle: beyond sustainability-designing for abundance. Straus and Giroux, New York Meadows DH, Meadows DL, Randers J, Behrens WW (1972) The Limits to Growth. A Report for the Club of Rome's Project on the Predicament of Mankind. Universe Books, New York Meadows DH, Randers J, Meadows DL (2006) Grenzen des Wachstums - Das 30-Jahre-Update. Signal zum Kurswechsel. Hirzel, Stuttgart

Midgley P (2011) Bicycle-sharing schemes: enhancing sustainable mobility in urban areas. http://www.cleanairinstitute.org/cops/bd/ file/tnm/13-bicycle-sharing.pdf. Zugegriffen: 9. Mai 2018

Mulhall D, Braungart M (2010) Cradle to cradle. Criteria for the built environment. Duurzaam Gebouwd, Nunspeet

Murdoch J (2000) Space against time: competing rationalities in plan- ning for housing. Trans Inst Br Geogr 25(4):503-519

Murphy DJ, Hall CAS (2011) Energy return on investment, peak oil, and the end of economic growth. Ann N Y Acad Sci 1219(1):52-72

Nadaï A, Van d Horst D (2010) Introduction: landscapes of energies. Landsc Res 35(2):143-155

North P (2010) Eco-localization as a progressive response to peak oil and climate change-a sympathetic critique. Geoforum 41(4):585-594

Osterhammel J (2011) Die Verwandlung der Welt. Eine Geschichte des 19. Jahrhunderts. $\mathrm{CH}$ Beck, München

Palensky P, Dietrich D (2011) Demand side management: demand re- sponse, intelligent energy systems, and smart loads. Trans Ind In- formatics 7(3):381-388

Palmas C, Siewert A, Haaren VC (2015) Exploring the decision-space for renewable energy generation to enhance spatial efficiency. En- viron Impact Assess Rev 52:9-17

Polanyi K (1957) The great transformation. Beacon Press, Boston Polimeni JM, Mayumi K, Giampietro M, Alcott B (2015)

The myth of resource efficiency. The jevons paradox. Routledge, London Prettenthaler FE, Steininger KW (1999) From ownership to service use lifestyle: the potential of car sharing. Ecol Econ 28(3):443-453

Quaschning V (2014) Regenerative Energiesysteme. Technologien, Berechnung, Simulation. Hanser, München Ramchurn SD, Vytelingum P, Rogers A, Jennings N (2011) Agentbased control for decentralised demand side management in the smart grid. https://eprints.soton.ac.uk/271985/2/ userfiles.soton. ac.uk_Users_nsc_mydesktop_271985ramchurn.pdf. Zugegriffen: 7. Febr. 2018 


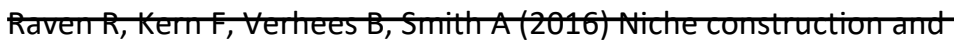
empowerment through socio-political work. A meta-analysis of six low-carbon technology cases. Environ Innov Soc Transitions 18:164-180

Resch G, Held A, Faber T, Panzer C, Toro F, Haas R (2008) Potentials and prospects for renewable energies at global scale. Energy Policy 36(11):4048-4056

Rifkin J (2013) The third industrial revolution. How lateral power is transforming energy, the economy, and the world. Pallgrave Mac- millan, Basingstoke

Roddis P (2018) Eco-innovation to reduce biodiversity impacts of wind energy: Key examples and drivers in the UK. Environ Innov Soc Transit 28:46-56

Rosa EA, Tuler SP, Fischhoff B, Webler T, Friedmann SM, Sclove RE, Shrader-Frechette K, English MR, Kasperson RE, Goble RL, Leschine TM, Freudenburg W, Chess C, Perrow C, Erikson K, Short JF (2010) Nuclear waste: Know-ledge waste? Science 329(5993):762-763

Sarkar S, Kern B (2008) Ökosozialismus oder Barbarei. Eine zeitgemäße Kapitalismuskritik. https://kritisches-netzwerk.de/forum/ oekosozialismus-oder-barbarei-eine-zeitgemaessekapitalismuskritik. Zugegriffen: 22. Mai 2018

Schmid A (2016) Neue Kontakte sorgen für Wirkungsgrad-Sprung bei Solarzellen. http://www.wiwo.de/technologie/green/tech/ photovoltaik-neue-kontakte-sorgen-fuer-wirkungsgrad-sprung- beisolarzellen/14639792.html. Zugegriffen: 8. März 2018

van der Schoor T, Bert Scholtens (2015) Power to the people: Local community initiatives and the transition to sustainable energy. Re- newable and Sustainable Energy Reviews 43:666675

Schumpeter JA (1912) Theorie der wirtschaftlichen Entwicklung. Duncker \& Humblot, Leipzig

Schumpeter JA (1994) History of economic analysis. Oxford Universi- ty Press, New York

Schumpeter JA (2009) Can capitalism survive? Creative destruction and the future of the global economy. Harper Perennial, New York Schwartzman D (2008) The limits to entropy: Continuing misuse of thermodynamics in environmental and Marxist theory. Sci Soc 72(1):43-62

Shove E (1998) Gaps, barriers and conceptual chasms: theories of technology transfer and energy in buildings. Energy Policy 26(15):1105-1112

Sieferle RP (1997) Rückblick auf die Natur. Eine Geschichte des Menschen und seiner Umwelt. Luchterhand, München

Sieferle RP (2001) The subterranean forest. Energy systems and the industrial revolution. The White Horse Press, Cambridgeshire

Solomon BD, Krishna K (2011) The coming sustainable energy transition: History, strategies, and outlook. Energy Policy 39(11): 7422-7431

Statista (2019) Prognostizierte statische Reichweite von Ressourcen. https://de.statista.com/statistik/daten/studie/152334/umfrage/statische-reichweite-von-ressourcen/. Zugegriffen: 11. März 2019

Stenzel T, Frenzel A (2008) Regulating technological change-The strategic reactions of utility companies towards subsidy policies

in the German, Spanish and UK electricity markets. Energy Poli- cy 36:2645-2657

Sturm B, Vogt C (2011) Marktversagen durch externe Effekte. In:

Strum B, Vogt C (Hrsg) Umweltökonomik - Eine anwendungs- orientierte Einführung. Springer, Heidelberg, S 17-40

Swyngedouw E (2010) Apocalypse forever? Post-political populism and the spectre of climate change. Theory Cult Soc 27(2-3): 213-232

Umweltbundesamt (UBA) (2016) Umweltschädliche Subventionen in Deutschland. https://www.umweltbundesamt.de/sites/default/ files/medien/479/publikationen/uba_fachbroschuere_ umweltschaedliche-subventionen_bf.pdf. Zugegriffen: 9. Juni 2018

Van d Bergh JCJM (2011) Environment versus growth-A criticism of "degrowth" and a plea for "a-growth". Ecol Econ 70(5):881-890

Van d Horst D (2007) NIMBY or not? Exploring the relevance of loca- tion and the politics of voiced opinions in renewable energy siting controversies. Energy Policy 35(5):2705-2714

Viebahn P, Soukup O, Samadi S, Teubler J, Wiesen K, Ritthoff M (2015) Assessing the need for critical minerals to shift the Ger- man energy system towards a high proportion of renewables. Re- new Sustain Energy Re-views 49:655-671

Walsh PR (2012) Innovation Nirvana or Innovation Wasteland? Identifying commercialization strategies for small and medium rene- wable energy enterprises. Technovation 32(1):32-42

Walter G, Gutscher H (2013) Generelle Befürwortung von Windkraftanlagen vor Ort vs. Befürwortung spezifischer Windkraftprojekte: Der Einfluss von Projekt- und Verfahrensparametern. Umweltpsy- chologie 17(2):124-144.

Walter K, Bosch S (2013) Intercontinental cross-linking of power sup- ply-calculating an optimal power line corridor from North Africa to Central Europe. Energy Sustain Soc 3(7). https://doi.org/10. 1186/2192-0567-3-14

Wangler LU (2013) Renewables and innovation: did policy induced 
structural change in the energy sector effect innovation in green technologies? JEnviron Plan Manag 56(2):211-237 Willis KJ, Bhagwat SA (2009) Biodiversity and climate change. Science 326(5954):806-880

World Commission on Environment and Development (WCED) (1987) Report of the World Commission on Environment and Devel- opment: Our common future. http://www.un-documents.net/our- common-future.pdf. Zugegriffen: 29 . Nov. 2018

Yenneti K, Day R, Golubchikov O (2016) Spatial justice and the land politics of renewables: Dispossessing vulnerable communities through solar energy mega-projects. Geoforum 76:9099

Zoellner J, Schweizer-Ries P, Wemheuer C (2008) Public acceptance

of renewable energies: Results from case studies in Germany. Energy Policy 36(11):4136-4141 